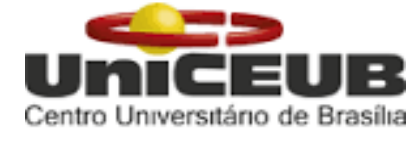

CENTRO UNIVERSITÁRIO DE BRASÍLIA - UniCEUB FACULDADE DE CIÊNCIAS DA EDUCAÇÃO E SAÚDE - FACES

\author{
PROGRAMA DE INICIAÇÃO CIENTÍFICA
}

LUCIENE DA SILVA GUEDES

HANID DE SOUSA VERSIANI

ESTUDO DA PREVALÊNCIA DE FATORES DE RISCO PARA SÍNDROME METABÓLICA EM UMA INSTITUIÇÃO DE ENSINO SUPERIOR

BRASÍLIA 


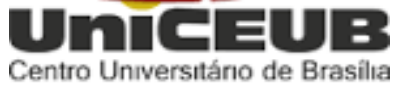

\section{LUCIENE DA SILVA GUEDES \\ HANID DE SOUSA VERSIANI}

\section{ESTUDO DA PREVALÊNCIA DE FATORES DE RISCO PARA SÍNDROME METABÓLICA EM UMA INSTITUIÇÃO DE ENSINO SUPERIOR}

\author{
Relatório final de pesquisa de Iniciação Científica \\ apresentado à Assessoria de Pós-Graduação e Pesquisa \\ pela Faculdade de Ciências da Educação e da Saúde - \\ FACES
}

Orientação: Prof. Msc. Vanessa Carvalho Moreira

\section{BRASÍLIA}




\section{DEDICATÓRIA}

Dedicamos o presente trabalho a Deus primeiramente, que nos deu força, inteligência e sabedoria para conseguirmos finalizar esse projeto.

À professora Vanessa Carvalho Moreira, por ter acreditado no nosso potencial, compreender todas as nossas dificuldades e limitações e nos impulsionar para o desenvolvimento acadêmico.

À nossa família e amigos, por nos apoiar nos momentos mais difíceis ao longo desse um ano de trabalho duro.

À preceptora de enfermagem do CAC, Carla e as diretoras do CA do mesmo curso, Ana Claudia Moraes Salomão e Sâmara Maria Vainauskas Barcelos, por toda disponibilidade e ajuda na captação dos voluntários, o nosso muito obrigado! 


\section{AGRADECIMENTOS}

Obrigada a todos os voluntários, pela disponibilidade em comparecer em jejum para as coletas de material biológico e pela confiança que depositaram na nossa pesquisa.

Aos funcionários do Labocien - UniCEUB, pela organização dos laboratórios e atenção sempre que precisamos usar as dependências na coleta dos dados e preparação das amostras.

Aos colegas e preceptores do CAC de biomedicina, pelo processamento das amostras e entrega dos resultados.

Aos secretários da FACES curso de biomedicina, em especial para Marcos, que sempre nos atenderam muito solicitamente sempre que precisamos.

Agradecemos enfim, imensamente, a todos que acreditaram conosco que esse projeto seria possível! 


\section{EPÍGRAFE}

"Existe uma força mais poderosa que a da eletricidade, do vapor e da energia atômica:

A VONTADE."

Albert Einstein 


\title{
Estudo da prevalência de fatores de risco para Síndrome Metabólica em uma Instituição de Ensino Superior
}

\author{
Luciene da Silva Guedes - UniCEUB, PIC institucional, aluno bolsista \\ luguedes1@gmail.com
}

Hanid de Sousa Versiani - UniCEUB, PIC institucional, aluno voluntário

hanidsmercyer@gmail.com

Vanessa Carvalho Moreira - UniCEUB, professor orientador

vanessa.moreira@uniceub.br

\section{Resumo:}

O desenvolvimento tecnológico e a escassez de tempo cada vez mais frequente vem alterando o modo de vida dos indivíduos, contribuindo para um estilo de vida sedentário, com alto nível de estresse emocional e alimentação desbalanceada. Isso acarreta alterações significativas na saúde das pessoas e contribui para a manifestação da Síndrome Metabólica (SM). A SM é caracterizada por um conjunto de alterações metabólicas que incluem a intolerância à glicose, hipertensão arterial sistêmica, aumento dos triglicerídeos e redução nos níveis de colesterol do tipo HDL. O objetivo deste trabalho é avaliar a prevalência de casos de Síndrome Metabólica em universitários da área da saúde do Centro Universitário de Brasília - UniCEUB. Trata-se de um estudo transversal experimental descritivo com 28 estudantes de 18 a 38 anos. Aos voluntários foram realizadas coletas de amostras de sangue para exames de glicose, hemoglobina glicada e perfil lipídico, além medidas antropométricas, cálculo do índice de massa corporal (IMC), aferição de pressão e aplicação de um questionário qualitativo para análise do perfil alimentar. O perfil corporal mais frequente, de acordo com o IMC, foi normal $(n=18)$ seguido de sobrepeso $(n=5)$ e obesidade $(n=1)$. De acordo com os critérios estipulados pela National Cholesterol Education Program's Adult Treatment Panel III (NCEP-ATP III), nenhum voluntário apresentou diagnóstico positivo para a SM. Com base nos dados aferidos no questionário, foi possível avaliar que, entre os hábitos alimentares, havia um alto consumo de carboidratos simples, doces, gordura saturada, frituras e sódio e baixo consumo de fibras, sais minerais, verduras, legumes e hortaliças. Ainda que não tenha sido observado a prevalência da SM na população estudada, é importante a realização de intervenções visando esclarecer, prevenir e orientar a população para o impacto da SM na saúde e os fatores de risco a ela associados, principalmente devido crescimento gradativo desta síndrome entre os jovens adultos.

Palavras-chave: Obesidade. Síndrome metabólica. Padrão alimentar. 


\section{SÚMARIO}

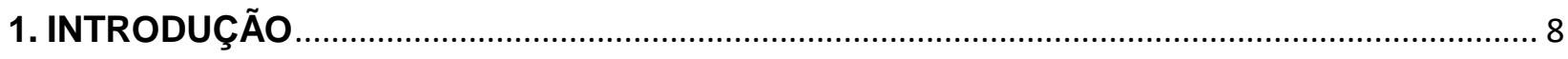

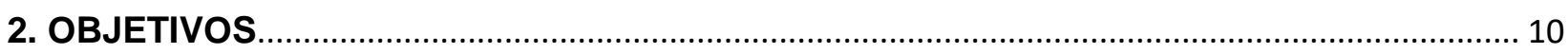

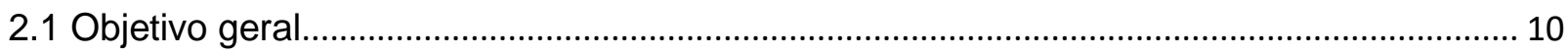

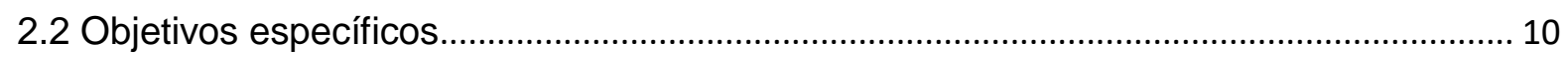

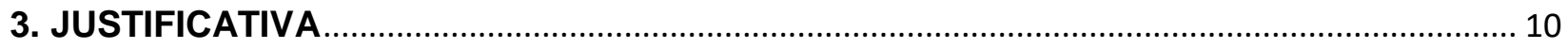

4. REVISÃO BIBLIOGRÁFICA E FUNDAMENTAÇÃO TEÓRICA ........................................... 11

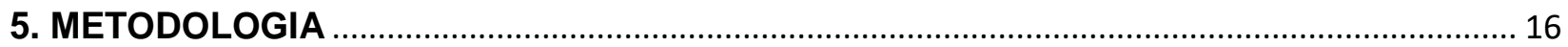

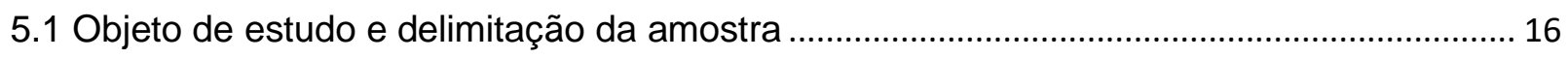

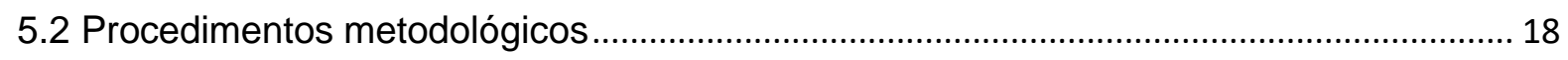

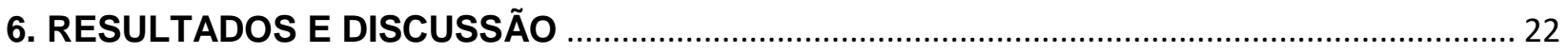

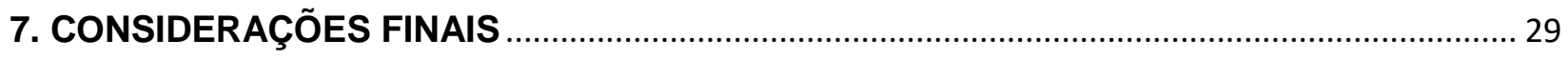

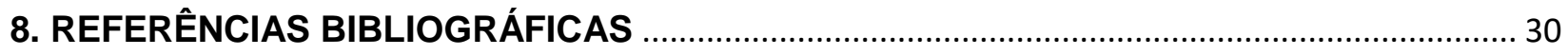

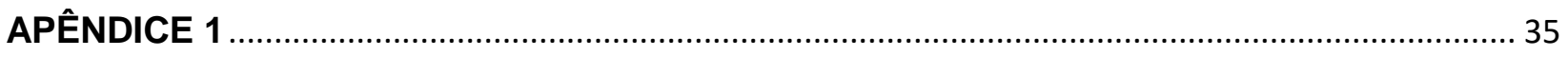

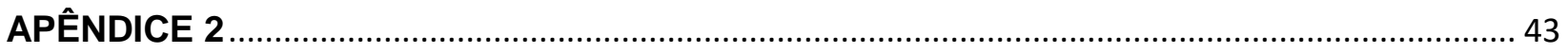

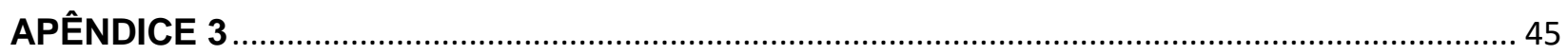




\section{INTRODUÇÃO}

As transformações ocorridas nos padrões socioeconômicos, culturais e a mudança no estilo de vida da população a partir da segunda metade do século $X X$, contribuíram para um expressivo processo de saúde-doença. Fatores como mudanças nos hábitos alimentares à base de uma dieta hipercalórica, sedentarismo e o estresse causado pela vida moderna tem influenciado a incidência de diversas doenças crônicas, como a hipertensão arterial, a obesidade e o diabetes mellitus. A correlação dessas alterações com o quadro clínico de resistência insulínica compõe a chamada síndrome metabólica (SM), ou seja, é um distúrbio metabólico que altera a homeostasia corporal, também definida como a "Síndrome da Civilização" (POZZAN et al.,2004; SALAROLI et al., 2007; GOTTLIEB, CRUZ, BODANESE 2008).

A SM é uma desordem multifatorial que promove em seus portadores, modificações metabólicas e cardiovasculares tais como: hipertensão arterial, hipertrigliceridemia, redução de colesterol HDL, intolerância a carboidratos, obesidade centrípeta e doenças cardiovasculares ateroscleróticas (DCVA). Esta síndrome é mais prevalente em indivíduos obesos e tem a resistência insulínica (RI) como um dos

principais fatores desencadeantes desta condição, contribuindo também para 0 surgimento da diabetes mellitus tipo 2 (DM2). Indivíduos com SM têm de 1,5 a 3 vezes maior risco para DCVA e 5 vezes maior risco para DM2 (BRANDÃO et al,2005; GELONEZE e TAMBASCIA,2006).

Em 1936, o médico inglês Himmsworth, notou em pacientes adultos dois tipos de diabetes mellitus. Em 1940, quatro anos depois, o francês Jean Vague descobriu dois tipos de obesidade. Apenas em 1980, depois de quarentas anos, que teóricos e cientistas esclareceram e descobriram a relação entre obesidade abdominal e os fatores típicos da SM. A partir daí foram vários estudos para definir e elucidar a determinada síndrome (GOODY-MATOS, 2011).

Atualmente a SM tem despertado grande interesse nos pesquisadores, sendo assunto abordado na comunidade acadêmica, em virtude dos seus fatores de risco (LAKKA et al., 2002). Esta síndrome tem prevalência global, que varia de acordo com a região e a composição da população estudada. Em geral, estima-se que um quarto da 
população adulta mundial tem SM. No Brasil, os estudos encontraram menor prevalência, mas significantes, variando de 6,7\% e 7,6\%. Sendo assim, a prevalência da síndrome metabólica difere de acordo com as regiões e populações,e também são relacionadas com as várias definições clínicas apresentadas (TURI et al., 2015). Há um aumento com o avançar da idade, atingindo o pico na oitava década de vida no sexo feminino e no sexo masculino na sexta década (PENALVA, 2008). Por isso a importância da detecção precoce da SM, entre outros fatores, pelo risco aumentado desses indivíduos portadores dessa alteração de desenvolverem doenças cardiovasculares, com alto índice de mortalidade. O diagnóstico em indivíduos mais jovens é imprescindível, pois permite o controle desses fatores evitando possíveis consequências que podem ser irreparáveis (CASTRO; MATO; GOMES, 2006).

No Brasil, pouco se sabe a respeito do panorama geral da SM, pois há uma escassez de estudos epidemiológicos relacionados à sua prevalência, destacando-se o estudo realizado em Vitória-ES que relatou o aumento gradativo da prevalência de SM em mulheres, e a relação entre doenças cardiovasculares e a presença precoce da SM em jovens (SALAROLI et al., 2007).Outro estudo realizado em São Luís-MA, numa população $\geq 13$ anos, onde a prevalência de $S M$ foi maior em ambos os sexos e o fator mais prevalente foi hipertensão arterial sistêmica (HAS) (BARBOSA et al, 2010). De acordo com um estudo realizado no Rio de Janeiro, com pacientes $\geq 18$ anos, onde se observou que a SM progride com o avançar da idade, entre os sexos não obtiveram resultados significantes, mas revelou uma associação positiva entre massa corporal e idade com a síndrome (LEÃO, BARROS, KOIFMAN, 2010). 


\section{OBJETIVOS}

\subsection{Objetivo geral}

Avaliar a prevalência de casos de Síndrome Metabólica (SM) em universitários da área da saúde do Centro Universitário de Brasília - UniCEUB, com base nos

critérios da Sociedade Brasileira de Endocrinologia, a fim de informar e prevenir a população estudada para os riscos de surgimento de doenças metabólicas crônicas.

\subsection{Objetivos específicos}

- Aplicar questionário para avaliar o perfil alimentar e sua relação com possíveis casos de síndrome metabólica na população estudada;

- Observar a prevalência de obesidade/sobrepeso entre os estudantes e sua relação com a manifestação da síndrome metabólica;

- Informar ao público alvo sobre a síndrome metabólica e suas consequências à saúde;

- Promover a conscientização do público jovem ao cuidado à saúde, prevenindo o surgimento de doenças crônicas.

\section{JUSTIFICATIVA}

No Brasil é observado um alto índice de doenças cardiovasculares, explicando o aparecimento precoce da Síndrome Metabólica na população brasileira, destacando a elevada prevalência em indivíduos mais jovens (SALAROLI et al., 2007).

O rastreio da Síndrome Metabólica é importante pois há um risco aumentado de desenvolver doenças cardiovasculares. A identificação precoce da SM é essencial para estratificar o risco geral do paciente e iniciar um tratamento imediato e adequado, com o intuito de controlar todas as alterações presentes e não desencadear consequências, que ás vezes é irreparável. A partir disso, nota-se que a síndrome metabólica está ligada ao estilo de vida, onde a falta de atividade física regular (sedentarismo), leva a obesidade central e, por conseguinte à hipertensão arterial e a resistência insulínica. 
Este conjunto de alterações pode levar o indivíduo a desenvolver a síndrome (CASTRO et al., 2006).

Outros fatores para o desenvolvimento da SM, de acordo com Cañete et al (2007), é o padrão alimentar do indivíduo. A alta ingestão energética, de ácidos trans, gordura saturada e ácidos graxos, baixo consome de fibras, dietas ricas em carboidratos refinados, com alto índice glicêmico, aparentemente influenciam no desenvolvimento da resistência insulínica e da obesidade. Segundo Lutsey et al., (2008) o consumo elevado de alimentos refinados, refrigerantes, frituras e carnes processadas, por exemplo, eleva a 18\% o risco de incidência da síndrome metabólica entre pacientes adultos.

Diante do exposto, é notória e importância de investigar, analisar e levantar dados sobre o perfil de jovens universitários quanto a manifestação ou predisposição à Síndrome Metabólica.

\section{REVISÃO BIBLIOGRÁFICA E FUNDAMENTAÇÃO TEÓRICA}

A síndrome metabólica (SM) teve suas origens em 1920, quando o médico Kylindemonstrou a associação da hipertensão, hiperglicemia e gota. Mais tarde,Vague em 1947, descreveu que a obesidade visceral era comumente associada às anormalidades metabólicas encontradas nas doenças cardiovasculares e diabetes mellitus tipo 2(DM2). Em 1965, um resumo foi apresentado na reunião anual da Associação Europeia para o Estudo do Diabetes, onde Avogaro e Crepaldi, descreveram novamente uma síndrome que inclui hipertensão, hiperglicemia e obesidade. A Conferência de Banting de 1988, ministrada por Gerald Reaven, médico endocrinologista do Departamento de Medicina Cardiovascular da Universidade de Stanford na Califórnia, também associou que alguns fatores de risco apareciam com frequência em determinados indivíduos e que estes também apresentavam ligeira sensibilidade à insulina. A essa condição ele designou o nome de Síndrome da Resistência à Insulina ou Síndrome X (KAUR, 2014).

Em 1998, naquele mesmo ano, a Organização Mundial da Saúde (OMS) determinou um critério de definição para esse quadro, incluindo nele a hipertensão 
arterial, dislipidemia, obesidade e microalbuminúria, chamando-o pela primeira vez de Síndrome Metabólica (SM). Em 2001, o National Cholesterol Education Program (NCEP) reuniu o $3^{\circ}$ Adult Treatment Panel (ATP III) outro critério de definição que não utilizasse o peso e a microalbuminúria, mas outros fatores de risco contemplados em outros sistemas. Em abril de 2005, foi publicada a I Diretriz Brasileira de Diagnóstico e Tratamento da Síndrome Metabólica com o apoio da Sociedade Brasileira de Cardiologia. Embora o critério adotado e mais seguido seja o da ATP III, alguns pesquisadores defendem que: a SM não esteja bem-definida e bem-caracterizada tornando-a confusa e ambígua; que o valor médico do diagnóstico ainda não é evidente e que por estas razões certos autores preferem chamar a SM de risco cardiometabólico (LUNA, 2006, O'NEILL; O'DRISCOLL, 2015).

Dessa maneira, a SM é um transtorno complexo representado por um conjunto de fatores de risco para doenças cardiovasculares, geralmente relacionados à deposição de gordura central e à resistência insulínica (RI). Contudo, não deve ser encarada como uma doença, mas sim como um complicado estado pré-patológico. $O$ número crescente de pessoas com obesidade e sobrepeso aumenta na mesma proporção que o número de pacientes com RI e portadores de SM. A obesidade por sua vez, pode ser definida como uma doença de etiologia multifatorial sendo dividida em dois fatores: endógenos - componentes genéticos, endócrinos, neuropsicológicos e metabólicos e exógenos - fatores externos como comportamentais, dietéticos e ambientais (CESARETTI e KOHLMANN JR, 2005; CAMPOS et al., 2006; MANA et al., 2006, REAVEN, 2011).

As grandes variações na susceptibilidade e idade de início em indivíduos com perfil de risco muito parecido, sugerem interação entre fatores genéticos e ambientais. É possível que a expressão de cada fator metabólico tenha seu próprio controle genético, o que influencia na resposta a diferentes exposições ambientais. Como exemplo temos uma gama de polimorfismos nos genes que afetam o metabolismo das lipoproteínas e que está associada ao agravamento da dislipidemia entre obesos. Como da mesma forma, uma predisposição genética à secreção de insulina pode aumentar a glicemia plasmática para níveis patológicos (KAUR, 2014). 
Estudos sugerem que a RI em conjunto com o aumento do tecido adiposo intraabdominal, podem ter um papel central na patogênese da SM, estando diretamente relacionado a vários transtornos do metabolismo inerentes a essa síndrome (PINEDA, 2008). Dentre eles pode-se citar: a elevação dos níveis plasmáticos de glicose (hiperglicemia) provocando o aumento da secreção de insulina (hiperinsulinemia) pelo pâncreas, o que pode gerar em longo prazo RI e DM2; a sensibilidade aumentada ao cortisol que também contribui para a hiperglicemia; o aumento dos níveis da angiotensina que pode acarretar risco de hipertensão; a elevação da secreção de interleucina-6 (IL-6) presente em processos inflamatórios e na lipólise, promovendo a formação de placas ateroscleróticas; a alta nos níveis de triglicerídeos (TG) que aumentam o risco cardiovascular por uma maior viscosidade sanguínea; a redução do colesterol HDL, de fundamental importância no transporte reverso do colesterol e que possui também influência anti-inflamatória, antioxidante e vasodilatadora e o fato de que o tecido adiposo visceral (TAV), ter maior sensibilidade à lipólise e apresentar uma elevada resistência à ação da insulina, acarretando numa maior demanda de glicerol e ácidos graxos aos hepatócitos, estimulando assim a gliconeogênese e aumentando a secreção de VLDL (lipoproteína de densidade muito baixa) (FERRARI, 2007; HERMSDORFF e MONTEIRO, 2004).

Todos os fatores envolvidos na patogênese da RI e da SM, levam aos efeitos metabólicos que caracterizam essa síndrome. Ademais, algumas sequelas metabólicas clínicas importantes são conhecidamente atribuídas a SM, como hiperglicemia e DM2, hipertensão, dislipidemia, doença hepática gordurosa não alcoólica, síndrome do ovário policístico, disfunção sexual masculina e apneia obstrutiva do sono. Contudo estudos associam uma conexão também com o câncer, mostrando um risco aumentado de mortalidade. Num grupo de 33.000 homens sem diagnóstico pré-existente de câncer, foi observado que aqueles que tinham diagnóstico de SM, por pelo ao menos 14 anos, tiveram um risco de $56 \%$ maior de mortalidade. Outro trabalho com mulheres apontou que as que tem maiores níveis de insulina, apresentaram maior risco de desenvolver câncer colorretal e endometrial (LAM, LeROITH, 2015).

Em relação à epidemiologia, a SM é uma manifestação patológica de relevância mundial presumidamente responsável por aumento expressivo de mortalidade por 
doença vascular. Sua prevalência é modulada por fatores hereditários e ambientais e é dependente da característica populacional e dos critérios de diagnóstico utilizados (MARCHI-ALVES et al, 2012). A falta de consenso para o critério de diagnóstico resulta em estimativas variadas no seu predomínio, contudo é possível observar resultados crescentes em países desenvolvidos e em desenvolvimento. Constata-se entre a população adulta mundial uma elevada prevalência, variando entre $25 \%$ a $35 \%$ e com maior frequência em mulheres, embora seja observado aumento conforme o avançar da idade e independente do sexo (LEÃO, BARROS, KOIFMAN, 2010, SBD, 2015). No entanto, no Brasil, as características epidemiológicas são pouco exploradas nos habitantes, não havendo revisão sistemática que defina a realidade da uma população tão miscigenada. Há apenas estudos em grupos específicos que podem refletir nos valores reais da comunidade (BORTOLETTO et al., 2014). Para exemplificar essa assertiva, Dutra et al (2007)investigou,no Distrito Federal, a prevalência da SM entre indivíduos maiores de 18 anos, que foi de $32 \%$ sem diferença de gênero.

No que tange ao diagnóstico, as diretrizes mais utilizadas no Brasil é ditada pela Diretriz Brasileira de Diagnóstico e Tratamento da SM que segue as recomendações do National Cholesterol Education Program's Adult Treatment Panel III (NCEP-ATP III), que utiliza dados clínicos e laboratoriais que podem ser obtidos de maneira facilitada, onde a presença de três dos cinco fatores é suficiente para diagnóstico da SM. A RI não é requisito básico para a confirmação da $S M$, mas está presente na maioria dos indivíduos, (PENALVA, 2008).

Entretanto, em 2006 o IDF (International Diabetes Federation) sugeriu que um único manual fosse seguido na prática clínica, pois a utilização de vários guidelines pelo mundo gera muitos estudos e divergências em relação ao diagnóstico preciso da SM. O IDF propõe para diagnóstico correto a medida da circunferência abdominal de acordo com os valores étnicos específicos (até dados mais específicos estarem disponíveis são utilizadas as medidas dos indivíduos do sul da Ásia para América Central e do Sul) mais os resultados positivos de dois dos cinco parâmetros também adotados pela NCEP/ATP III (IDF, 2006).

Estudo brasileiro, realizado entre adolescentes em 2014, demonstrou que a utilização da medida da circunferência do pescoço (CP) também pode ser utilizada 
como parâmetro para diagnóstico da SM, como a da circunferência da cintura (CC). Segundo os autores, a CP em comparação com a CC tem muito mais vantagens. Entre as principais descritas estão a facilidade de aplicação e o baixo custo, viabilizando sua utilização em serviços de Saúde Pública e em estudos epidemiológicos. Em contrapartida a única desvantagem é que não apresenta valores internacionais de referência que possibilitem sua ampla utilização (SILVA, 2014).

Quanto aos fatores de risco relacionados à SM, o nível de atividade física, os hábitos alimentares, predisposição genética e a obesidade são fatores de relevância que influenciam o desenvolvimento da síndrome e dos seus componentes (SINGH et al., 2013, ECKEL, 2005). Nesse sentido, a dieta e a atividade física são determinantes no peso corporal e podem favorecer o desenvolvimento da SM, da obesidade e da alteração do índice de massa corpórea (IMC). Apesar da grande influência do IMC na $\mathrm{SM}$, estudos apontam que mesmos os indivíduos com IMC normal ou taxa de gordura corporal total baixa podem apresentar a referida síndrome. Isso seria explicado pelo fato do aumento ainda que quase exclusivo da adiposidade visceral, mensurado pela circunferência abdominal, ser proporcional à elevação da RI (RIBEIRO FILHO et al., 2006).

Uma vez diagnosticada a SM, o tratamento é focado em medidas não medicamentosas e medicamentosas. As medidas não medicamentosas são baseadas na adoção de uma dieta balanceada individualizada e atividade física regular e contínua. Por sua vez, o tratamento medicamentoso consiste em normalizar os parâmetros alterados presentes nos pacientes com a SM. Ambos são direcionados para perda de peso e da gordura visceral, com o objetivo de normalização da pressão arterial (PA), correção da dislipidemia e hiperglicemia. (BRANDÃO et al., 2005,FERREIRA, 2016).

Nesse sentido, medidas educacionais de saúde e bem-estar, focadas em um padrão alimentar saudável, atividade física regular e melhoria do estilo de vida em geral devem ser incentivadas. Somente a atuação com o intuito de prevenir ou eliminar os componentes da SM, poderá garantir de forma efetiva um estilo de vida sadio e, assim, corroborar favoravelmente na prevenção de enfermidades cardiovasculares, diabetes, entre várias outras patologias graves (KUSCHNIR et al., 2016). 


\section{METODOLOGIA}

Foi realizado uma pesquisa básica quantitativa por meio de um estudo transversal experimental descritivo dos estudantes da área da saúde do Centro Universitário de Brasília - UniCEUB.

\subsection{Objeto de estudo e delimitação da amostra}

O objeto de estudo é a avaliação da presença de síndrome metabólica em estudantes da saúde, uma vez que, pelo conhecimento adquirido durante a graduação, espera-se apresentar maior consciência dos riscos que os distúrbios metabólicos podem causar à saúde.

Os critérios de inclusão adotados foram:

- $\quad$ Estar devidamente matriculado em cursos da saúde do UniCEUB;

- $\quad$ Apresentar idade igual ou superior a 18 anos;

- Que responda o questionário alimentar (apêndice 1) para levantamento de dados.

- $\quad$ Ter assinado o "Termo de Consentimento Livre e Esclarecido" (apêndice 2)

- Não apresentar qualquer problema de saúde de natureza mental ou física, sendo dotados de capacidade para determinar sua participação ou não nos experimentos.

Quanto aos critérios de exclusão, aqueles que não puderam ser incluídos no presente estudo são:

- $\quad$ Idade inferior a 18 anos;

- Que não cumpriu todas as etapas discriminadas nesse estudo;

- $\quad$ Que não autorize a utilização de seus dados por meio da assinatura do "Termo de Consentimento Livre e Esclarecido".

Foram selecionados por meio dos critérios de inclusão e de forma voluntária estudantes do UniCEUB graduandos na área da saúde, preferencialmente nos cursos de Biomedicina, Enfermagem e Educação Física. Os participantes realizaram exames de sangue como glicose pelo método enzimático com hexoquinase, hemoglobina 
glicada pelo método imunoturbidimetria e perfil lipídico pelos métodos (colorimétrico enzimático, colorimétrico enzimático homogêneo e fórmula de Friedewald e Martin), medidas antropométricas, aferição de pressão e análise do perfil alimentar. Importante salientar que para os exames, os voluntários encontravam-se em jejum.

Os doadores assinaram o termo de consentimento livre e esclarecido (TCLE) (apêndice 2) e nenhum indivíduo foi compelido, coagido ou forçado a assinar o termo, obtendo plena autonomia para concordar ou não sobre a doação voluntária. Todos os participantes receberam por e-mail os resultados do exame de sangue.

A anamnese dos pacientes e a coleta de sangue foram realizadas no laboratório multidisciplinar do Labocien, localizado na Faculdade de Ciências da Educação e da Saúde - FACES do UniCEUB, bloco 9 - campus Asa Norte - SEPN 707/907, BrasíliaDF, seguindo os padrões de biossegurança necessários para manipulação de material biológico. As análises laboratoriais foram processadas no laboratório-escola de análises clínicas do UniCEUB, localizado na quadra 1, do Setor Comercial Sul.

De acordo com as normas éticas, este trabalho foi submetido e aprovado pelo Comitê de Ética em Pesquisa do Centro Universitário de Brasília, sobre o número $1.906 .906 / 2017$ 


\subsection{Procedimentos metodológicos}

Os procedimentos metodológicos seguiram o fluxograma descrito abaixo.

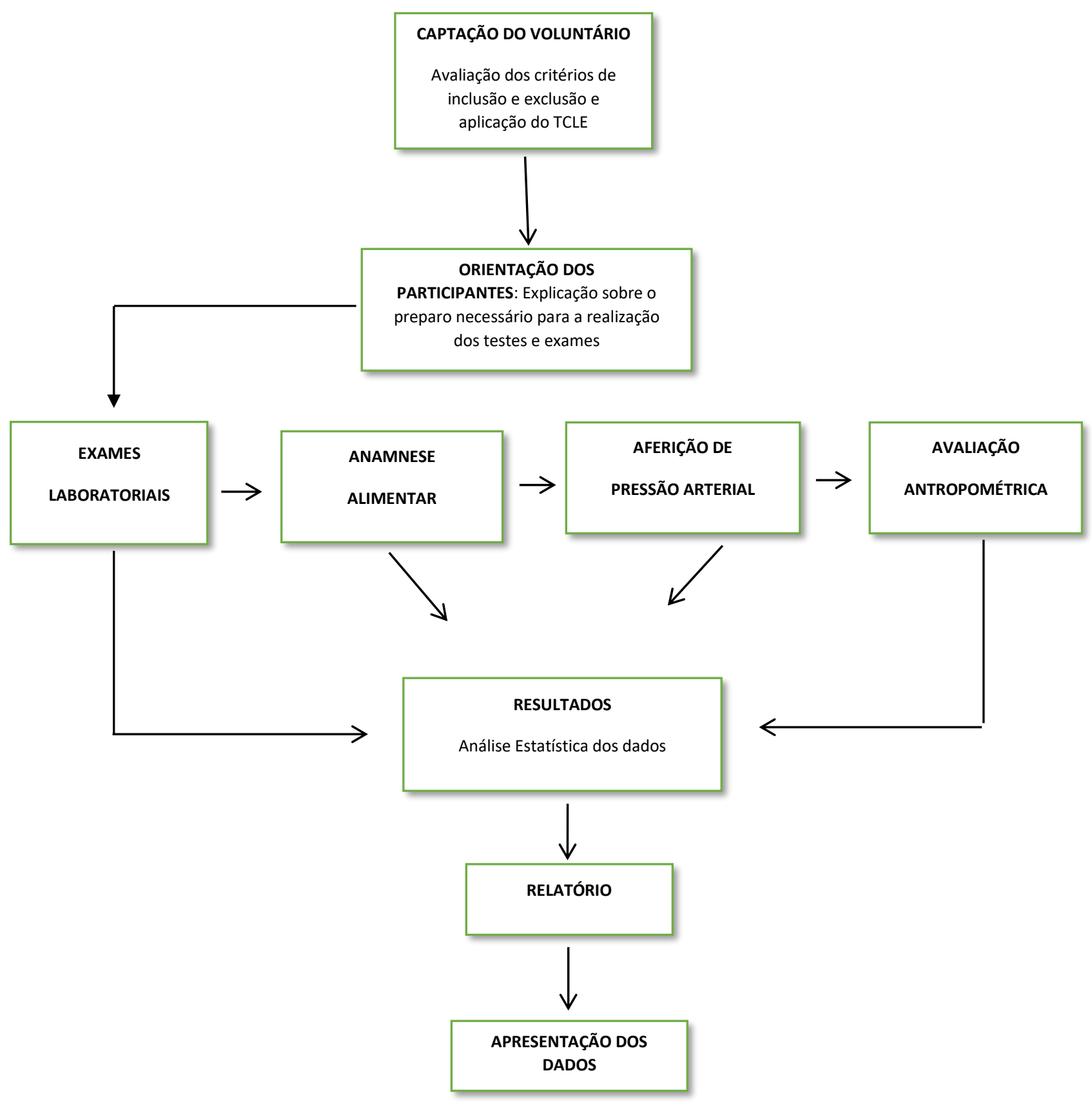


Para a realização dos exames bioquímicos utilizou-se amostras de sangue, da veia na região da fossa cubital, visando a obtenção do soro. O material foi coletado, por profissionais treinados, em tubos contendo gel separador para dosagem de perfil lipídico e outro tubo contendo fluoreto de sódio para análise da glicemia. Para a coleta foram utilizadas agulhas descartáveis, seguindo o procedimento padrão e estabelecido na literatura. O voluntário necessitava de jejum de 12 horas. Por se tratar de manipulação de material biológico com risco em potencial, a fim de evitar qualquer tipo de contaminação todos os equipamentos de proteção individuais necessários (EPI) foram utilizados.

Quantos aos hábitos alimentares aplicou-se um questionário qualitativo (apêndice 1). Este elemento é fundamental para observarmos se a alimentação do indivíduo o predispõem a manifestar a síndrome metabólica.

A aferição da pressão arterial seguiu o protocolo de diretrizes clínicas, onde a medida da pressão arterial (PA) é realizada pelo método indireto através de técnica auscultatória com uso de esfigmomanômetro de coluna de mercúrio ou aneroide, devidamente calibrado. Todo o processo não confere qualquer risco ao paciente, que foi informado a respeito das recomendações necessárias para a aferição da PA. Os resultados desse procedimento são importantes para o estudo, pois determinam se 0 voluntário apresenta um dos fatores de risco para a Síndrome Metabólica, que é o aumento da PA.

Para a avaliação antropométrica esta foi iniciada com o cálculo do índice de massa corporal (IMC) do indivíduo dividindo-se o peso (em $\mathrm{Kg}$ ) pelo quadrado da altura (em metros). O resultado revela se o peso está dentro da faixa ideal, abaixo ou acima do desejado. É utilizado na prática clínica para diagnóstico e classificação da obesidade, orientação nutricional, avaliação física, verificação de fatores de risco cardiovasculares e síndrome metabólica.

Em seguida, realizou-se a avaliação das dobras cutâneas seguindo o método de Jackson e Pollock (apêndice 3), partindo inicialmente pela aferição das circunferências corporais por meio de uma fita métrica de plástico graduada de 0 a $150 \mathrm{~cm}$ com intervalos de $0,5 \mathrm{~mm}$. As medidas foram avaliadas nos seguintes locais anatômicos: 
a) Peitoral - Medida diagonal tirada a meio caminho entre o mamilo direito e a parte superior do peitoral;

b) Abdominal - Medida vertical tirada a 2,5 cm à direita do umbigo;

c) Coxa - Medida vertical tirada entre a patela e a dobra inguinal;

d) Tríceps - Medida vertical tirada ao meio entre a parte superior do ombro e o cotovelo;

e) Subescapular - Medida diagonal tirada na parte superior das costas, imediatamente abaixo das omoplatas, a um ângulo de 45;

f) Supra ilíaco - Medida diagonal tirada acima da crista ilíaca anterior superior;

g) Axila - Medida vertical tirada diretamente no centro da axila, ao nível do mamilo;

h) Pescoço - Medida na menor circunferência do pescoço, logo acima da proeminência laríngea.

Posteriormente, foram avaliadas as medidas com adipômetro. Todas as medidas foram feitas do lado direito do corpo e a dobra cutânea tomada entre o polegar e o indicador esquerdos. Durante o procedimento, faz-se necessário o cuidado de não incluir o tecido muscular (em caso de dúvida, recomenda-se a execução de uma contração antes de ser efetuada a medida). Após dois segundos de compressão, faz-se a leitura da medida. Para a garantia da qualidade dos dados obtidos, o procedimento foi repetido três vezes em cada medida (alternando) buscando-se uma boa correlação entre elas (máximo 5\% de variação) e tirar a média. Se houve variação maior que 5\%, uma nova série de três medidas foi realizada. As medidas coletadas foram:

a. Dobra tricipital: medida vertical da parte posterior do braço a meio caminho entre o acrômio e a linha radial e, estando com o braço relaxado;

b. Dobra subescapular: medida diagonal $\left(45^{\circ}\right)$ abaixo do ângulo inferior da escápula;

c. Dobra bicipital: medida vertical na mesma localização do tríceps, só que anteriormente;

d. Dobra Peitoral: Homens: medida diagonal do ponto médio entre a linha anterior e o mamilo. Mulheres: medida diagonal no terço proximal do mesmo seguimento;

e. Dobra Axilar Média: medida diagonal na linha de interseção entre a linha axilar e do ílio ao nível do processo xifoide. Estando os braços levantado a $90^{\circ}$;

f. Dobra supra ilíaca: medida diagonal seguindo o ângulo natural da crista ilíaca; 
g. Dobra. Abdominal: medida vertical localizada aproximadamente a $5 \mathrm{~cm}$ da cicatriz umbilical. Ao lado direito e não colocando o compasso no interior da cicatriz umbilical;

h. Dobra da Coxa: medida vertical entre a linha inguinal e a borda superior da patela, estando a perna levemente flexionada;

i. Dobra da Perna: medida vertical com o joelho flexionado a $90^{\circ}$ e relaxado na parte medial maior de circunferência.

Os voluntários foram orientados a comparecer com vestimenta apropriada no momento da verificação dos parâmetros antropométricos: short e sem camisa para homens e short e top para mulheres, entretanto, os pesquisadores tomaram o cuidado de evitar a exposição dos participantes. Os resultados dos procedimentos descritos anteriormente são importantes para o estudo, pois determinam se o voluntário apresenta índices de gordura corporal e o IMC elevados, sendo um dos fatores de risco para a obesidade e consequentemente para a síndrome metabólica.

Os dados selecionados foram registrados em uma planilha visando identificar, determinar, caracterizar e conhecer as temáticas tratadas através de um quadro esquemático.

Para o cálculo da prevalência foi utilizada a seguinte fórmula:

$\frac{\mathrm{N}^{\circ} \text { de indivíduos afetados }}{\text { POPULAÇÃO TOTAL }}$

As análises foram realizadas empregando-se planilhas do Excel, seguida pela produção de elementos gráficos que demonstram de forma fidedigna, o que foi observado. 


\section{RESULTADOS E DISCUSSÃO}

Foram avaliados 28 estudantes (7 homens e 21 mulheres) dos cursos de Biomedicina $(n=10)$, Enfermagem $(n=17)$ e Educação Física $(n=1)$, com idade compreendida entre 18 a 38 anos (tabela 01 ).

Tabela 1: Caracteríticas dos estudantes.

\begin{tabular}{lc}
\hline Idade (anos) & 18 a 38 \\
\hline Sexo (F/M) & $21 / 7$ \\
Biomedicina & 10 \\
Enfermagem & 17 \\
Educação Física & 1 \\
Total & 28 estudantes \\
\hline
\end{tabular}

F: feminino; M: masculino.

Quanto ao perfil corporal, adquirido por meio do cálculo do índice de massa corporal(IMC), observamos que 18 indívíduos estão normais (entre 18,5 e 24,9), cinco com sobrepeso (entre 25 e 29,9), um com grau de obesidade (entre 30 e 40) e quatro indivíduos abaixo do peso (menor que 18,5), este último todos do sexo feminino(figura 1). O valor da circunferência abdominal, um dos elementos importantes para 0 diagnóstico da SM, mostrou-se fora dos valores considerados de risco (homens: cintura acima de $102 \mathrm{~cm}$ e mulheres: acima de $88 \mathrm{~cm}$ ) em todos os indivíduos analisados. 


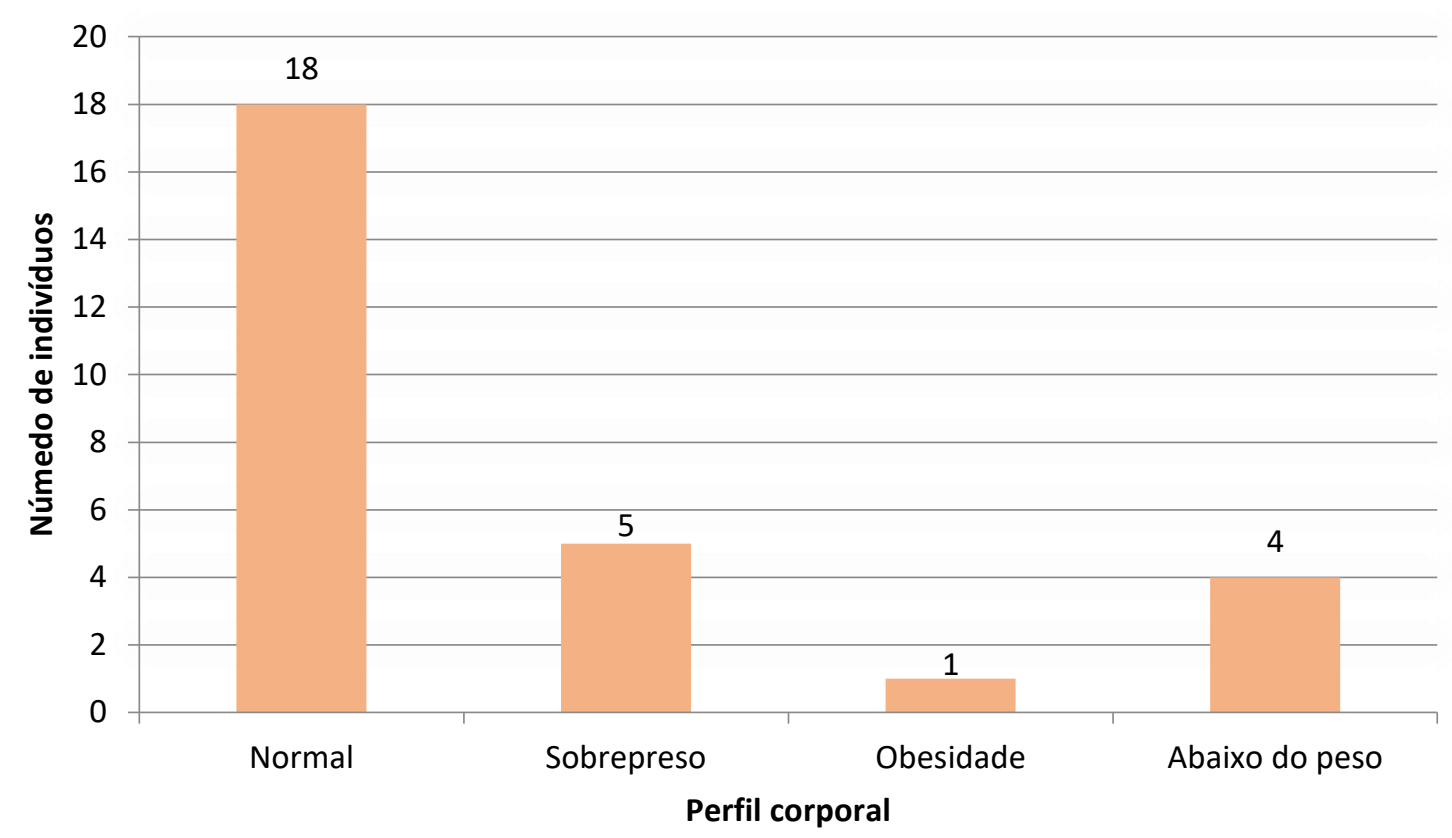

Figura 1. Perfil corporal dos universitários conforme índice de massa corporal (IMC)

Segundo a Organização Mundial de Saúde (OMS), o índice de massa corporal (IMC) é recomendado como indicador nutricional e frequentemente utilizado em estudos epidemiológicos, na área clínica e em saúde pública. Há uma forte ligação entre obesidade abdominal e as doenças cardiovasculares, sendo essa obesidade central um dos fatores determinantes para a síndrome metabólica (SM). Desse modo, a relevância da obesidade está fundamentada na literatura e mostra sua relação com doenças crônicas não transmissíveis relacionadas ao IMC (CHOCAIR, 2016).

Em contrapartida, Cao et al. (2014), em estudo retrospectivo de outros 51 trabalhos, demonstraram a relação do baixo peso e a SM e o risco de morte por qualquer causa. Segundo os pesquisadores, a obesidade mórbida aumentou a chance de morte em cinco anos em $30 \%$, a obesidade em $20 \%$ e o baixo peso em $80 \%$. Isso se deve por alguns fatores como consumo excessivo de drogas ou álcool, tabagismo, desordens psiquiátricas e/ou desnutrição, aspectos que fazem parte do estilo de vida de muitos jovens na faixa etária estudada. Os autores acreditam que tais resultados 
demonstram que restringir muito a alimentação ou se exercitar em demasia é tão danoso quanto estar acima do peso.

Todavia, a circunferência abdominal é ainda mais importante, pois traduz melhor a obesidade visceral que, por sua vez, está intimamente correlacionada a SM e o risco cardiovascular, mesmo em indivíduos com IMC normal, mas com cintura abdominal alterada (CHOCAIR, 2016). Além disso, o aumento da gordura abdominal e do IMC elevam a glicemia de jejum, os níveis séricos dos triglicerídeos, diminuem os de HDLcolesterol e aumentam a pressão arterial (REZENDE, 2006).

A manifestação da SM entre os estudantes foi analisada com base nos critérios da NCEP/ATP III que consiste na presença de três dos cinco fatores descritos a seguir: glicemia maior ou igual a $100 \mathrm{mg} / \mathrm{dL}$ ou em tratamento para hiperglicemia; HDLcolesterol em homens menor que $40 \mathrm{mg} / \mathrm{dL}$ e mulheres menor que $50 \mathrm{mg} / \mathrm{dL}$ ou ambos em tratamento para HDL baixo; triglicérides maior ou igual a $150 \mathrm{mg} / \mathrm{dL}$ ou em tratamento para triglicérides elevado; circunferência abdominal maior ou igual a $102 \mathrm{~cm}$ para homens e maior ou igual a $88 \mathrm{~cm}$ para mulheres e hipertensão maior ou igual 130 x $85 \mathrm{mmHg}$ ou em tratamento para HAS.

Diante dos dados coletados, a prevalência da SM em universitários da área da saúde foi de zero. Dos parâmetros analisados apenas três indivíduos (10\%) apresentaram triglicerídeos alterado (VR: $\geq 150 \mathrm{mg} / \mathrm{dL}$ ) e nove indivíduos(32\%) valores baixos de HDL-colesterol (VR: homens $<40 \mathrm{mg} / \mathrm{dL}$ e mulheres $<50 \mathrm{mg} / \mathrm{dL}$ ).

No Brasil, a frequência da SM é desconhecida em várias regiões e pouco estudada em diferentes populações. Porém estudos brasileiros realizados com universitários nos estados do Piauí (SILVA et al., 2014) e do Maranhão (CARVALHO et al., 2015, BARBOSA et al., 2016), corroboram com estudos internacionais também com alunos de nível superior que foram realizados nos EUA (MORRELL; COOK; CAREY, 2013, MORRELL et al., 2014, DALLECK; KIELLAND, 2012), México (VELÀZQUEZ; HERRERA; TAMAYOI, 2013) e Índia (USHA; CHANDRIKA; SHETTY, 2014).

De acordo com os dois estudos do Maranhão que utilizaram 968 voluntários cada um, a SM foi mais prevalente em homens, enquanto que no Piauí, com um número de 550 estudantesanalisados, esta foi maior em mulheres (CARVALHO et al., 2015, 
BARBOSA et al., 2016, SILVA et al., 2014). Não há consenso entre os estudos sobre essa prevalência da SM entre os gêneros, entretanto nos estudos citados foi observado que os homens têm maior quantidade de alterações dos parâmetros, possivelmente pelos hábitos de vida (SILVA; SALVO, 2011). Porém essa relação se inverte com o avançar da idade devido fatores hormonais femininos e o hipometabolismo (OTT et al., 2011).

Embora nenhum participante tenha manifestado características clínicas para o diagnóstico da SM é importante discutir sobre a alteração no perfil lipídico. O TG quando consumidos em excesso, se acumula nas células e se deposita como placas de ateroma causando elevado risco de doença arterial coronarianas (DAC). Além disso, a hipertrigliceridemia aumenta o risco de trombogênese por interagir com fatores de coagulação e plaquetas. Já o HDL- colesterol é responsável pelo transporte reverso, leva esse acúmulo para o fígado, desse modo, seus baixos níveis também são traduzidos como risco cardiovascular (SBC, 1994). Ressalta-se que estamos falando de uma população jovem que pode apresentar sinais de DAC e outras comorbidades crônicas precocemente, dessa maneira é vital reforçar a importância do diagnóstico prematuro para se reduzir o risco de menor sobrevida (SILVA et al., 2014).

Alterações significativas desses parâmetros também foram observados por Barbalho et al (2009), em estudo com 57 alunos de uma universidade pública em Marília-SP.Nos estudos realizados no México e índia, com 190 e 90 participantes respectivamente, os níveis de HDL baixo foram de $40 \%$ e $50 \%$, enquanto neste foi de $32 \%$. Contudo corrobora com a alteração nesse parâmetro no perfil lipídico de parte da população mundial (VELÀZQUEZ; HERRERA; TAMAYOI, 2013, USHA; CHANDRIKA; SHETTY, 2014). Em relação aos níveis de triglicerídeos (TG), nos três estudos Americanos, com 207, 360 e 1610 estudantes, os níveis de TG estavam aumentados em cerca de $40 \%$ dos voluntários, achado também presente neste trabalho em $10 \%$ dos adultos jovens (DALLECK; KIELLAND, 2012, MORRELL; COOK; CAREY, 2013, MORRELL et al., 2014).

Ademais,Dutra et al. (2012) e Cruz et al. (2014),apontam que o maior nível socioeconômico e de escolaridade (12 anos ou mais de estudo) são protetores contra a SM e seus fatores de risco em relação as mulheres, o que também foi percebido por 
este estudo.A maioria dos voluntários que tiveram algum parâmetro alterado foram predominantemente do sexo masculino. Em contrapartida, para Barbosa et al. 2016, foi observado que os universitários de instituições privadas tiveram maior prevalência de SM e associações, do que os das públicas.

Os resultados apresentados podem estar relacionados também ao fato de que entre os participantes do estudo, $75 \%$ eram do sexo feminino. Essa tendência de maior participação feminina comparada à masculina foi descrita por Azambuja, Farinha e Santosa 2015. As mulheres habitualmente não somente se preocupam mais com sua saúde em geral, como também são mais receptivas em aderir aos tratamentos propostos e à mudança de hábitos.

Quanto ao perfil alimentar dos estudantes, foi possível observar um alto consumo de carboidratos simples $(n=27)$ e doces $(n=20)$, gordura saturada $(n=21)$ e frituras $(n$ $=20)$, embutidos $(n=20)$ e multiprocessados $(n=23)$, e sódio $(n=24)$. Quanto a proteína animal, o consumo foi de alto a moderado $(n=22)$. Já o consumo de fibras ( $n$ $=21)$, sais minerias $(n=19)$, verduras, legumes e hortaliças $(n=19)$ foi extremamente baixo. Todos os dados estão representados na figura 2 .

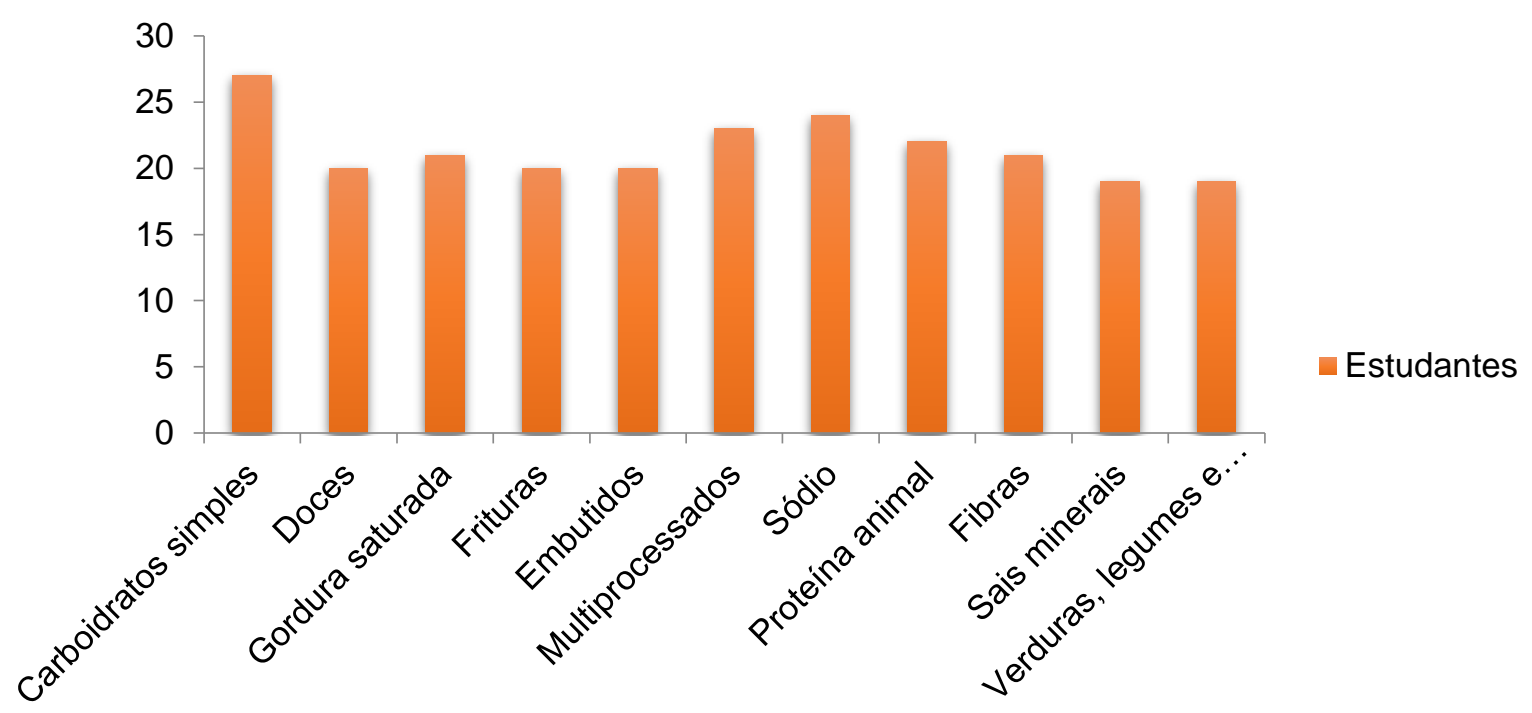

Figura 2: Perfil alimentar dos estudantes. 
Estudos evidenciaram que estudantes universitários constantemente desenvolvem hábitos alimentares inadequados (FEITOSA, 2010; PAIXÃO, 2010). Para alguns estudantes, é no período acadêmico o primeiro momento de suas vidas em que esses indivíduos jovens terão que responsabilizar-se por sua alimentação, administração de suas finanças e moradia, o que resulta no consumo frequente de lanches rápidos, refeições nutricionalmente desequilibradas e consequentemente omissão das principais refeições. Assim, o padrão alimentar adquirido nessa época, em vários casos, é preservado ao longo da vida (FOCHAT et al., 2016). O consumo alimentar influencia na SM, refletindo sobre o perfil lipídeo-lipoproteico plasmático, pressão arterial e gordura corporal, fortemente influenciados pelo consumo de produtos/alimentos açucarados e ricos em gordura (MARTINI; BORGES; GUEDES, 2014).

Em estudo realizado com 278 estudantes, observaram também que a dieta desses indivíduos eram hiperenergética, hiperproteica, e hiperglicídica, o que pode compor um fator de risco, a longo prazo, para a saúde (FOCHAT et al., 2016). Os carboidratos de alto índice glicêmico estão relacionados como fator para 0 desenvolvimento da obesidade e o alto consumo de proteínas pode resultar em diurese e desidratação, sendo que a longo prazo pode sobrecarregar a função renal e o aumento do trabalho hepático. Diversos estudos comprovam que o consumo de frutas, legumes e verduras possuem efeito protetor em alguns fatores como circunferência abdominal alterada, hiperglicemia e a presença da SM (RAMOS et al., 2013; ESMAILLZADEH et al., 2006; CASTANHO et al., 2013). Como observamos no presente estudo e em outros citados, o consumo desses alimentos é baixo entre os universitários. Dessa forma, é recomendado aos estudantes o aumento na ingestão de peixes, verduras, legumes, cereais integrais, frutas e outros alimentos ricos em fibras e sais minerais para prevenir o desenvolvimento da SM.

A necessidade de abordamos mais sobre a SM e informar à população quantos aos riscos dessa alteração e aos problemas de saúde que ela pode acarretar é notória.

De acordo com a OMS (2017), a quantidade de pessoas com diabetes aumentou de 108 milhões em 1980 para 422 milhões em 2014. Sendo que em 2015, aproximadamente de 1,6 milhões de óbitos foram diretamente ocasionados pelo 
diabetes e em 2012, foram atribuíveis a alta glicemia 2,2 milhões de mortes. A diabetes do tipo 2 abrange a maioria dos casos de diabetes no mundo, resultado em grande parte da falta de atividade física e excesso de peso corporal. A OMS visa que em 2030 a diabetes será a sétima principal causa de morte.

Para a prevenção da SM faz-se necessária uma mudança nos hábitos, como prática regular de exercícios, adoção de uma dieta mais saudável e equilibrada, controlar o peso e a pressão arterial, como também o controle do estresse e ansiedade. As crianças, adolescentes e jovens adultos tem no sedentarismo um dos principais vilões para o surgimento precoce de inúmeras condições pré-patológicas, como a SM. As futuras complicações podem ser catastróficas se não forem inseridas medidas de intervenção preventiva. Com isso, é necessário identificar indivíduos com maior risco para não desenvolver complicações futuras (SBD, 2014; VELAZQUEZ, HERRERA, TAMAYO, 2013).

Feoli e Gottlieb 2016,apontam que recursos tecnológicos estão sendo aliados de profissionais e pacientes por uma melhor qualidade de vida, com a utilização de tecnologia de informação e comunicação (TICs) através de jogos e aplicativos. Essa tem sido uma tendência crescente em âmbito mundial, pois a popularização de tablets e smartphones permitiram que aplicativos e programas a favor de práticas saudáveis ganhassem força. A junção das áreas da saúde, informática e comunicação convergem para um único sentido: auxiliar e instruir os jovens a cuidar melhor da saúde, com uma linguagem objetiva e acessível e práticas lúdicas. O uso de tecnologias compatíveis com o cotidiano e que desperte o interesse dos jovens, por meio de jogos e brincadeiras, aponta ser um importante aliado também contra os fatores predisponentes da SM.

As políticas públicas adotadas no Brasil têm sido voltadas, em sua maioria para a carência nutricional da população, visto que na atualidade ainda há casos de desnutrição, que são na maioria casos de carências específicas de vitaminas e minerais. O brasileiro sofre cada dia mais influência cultural de países desenvolvidos, destacando-se a presença dos hábitos alimentares dos nortes americanos. Em sua maioria tendo acesso a produtos processados e abandonando quase que totalmente uma alimentação saudável, repleta de frutas, verduras e legumes, aumentando o 
número de indivíduos com sobrepeso e obesidade. O país tem empenhado cada vez mais em políticas voltadas para a alimentação e nutrição. Mas ainda há escassez, em alguns municípios, de introdução do sistema de vigilância alimentar e nutricional, que visa auxiliar aos gestores públicos com um mapa mais específico do estado nutricional da população (EICKHOFF et al., 2012).

Embora os achados deste trabalho sejam relevantes e apresentem contribuição científica, a pouca adesão dos alunos dificultou a execução da pesquisa. Foi possível observar uma limitação em relação ao número amostral inicial de 33 voluntários, que foi reduzido para 28 , pois alguns não cumpriram todas as etapas discriminadas e foram excluídos. A prevalência zero, apesar de alguns participantes apresentarem um ou dois parâmetros positivos para a SM, pode ser entendida também pelo reduzido número amostral uma vez que a prevalência da SM é crescente entre a população mundial, incluindo adultos jovens universitários. Assim, recomenda-se estudos futuros com amostra mais representativa, bem como a utilização de questionário alimentar quantitativo e qualitativo, para se obter um melhor perfil alimentar e sua contribuição para a manifestação da SM. Outro ponto relevante, é a análise do perfil corporal ao longo da graduação onde observa-se um ganho de peso considerável entre os universitários quando comparado início e fim da graduação.

\section{CONSIDERAÇÕES FINAIS}

A prevalência da SM não foi relevante entre os estudantes universitários analisados nessa pesquisa, no entanto, alguns dos indivíduos possuem componentes presentes, aumentando as chances do aparecimento do distúrbio no futuro. Além disto, são estudantes pertencentes à área da saúde, o que pode significar maior conhecimento sobre os componentes da SM e os indicadores de saúde. É devido salientar que esses universitários serão agentes de saúde e formadores de opinião para a comunidade em que atuarem profissionalmente. Estudos de intervenção podem ser realizados para incentivar hábitos de vida saudáveis a todos os universitários e a população, independente da área de formação e período em que se encontram no curso. Incrementar no dia-a-dia atividade física para combater o sedentarismo e a 
obesidade associadas a padrões alimentares saudáveis podem ajudar a prevenir o desenvolvimento da SM e seus fatores de risco.

Ainda há poucos estudos definindo a relação entre a SM e estudantes no Brasil, bem como na América Latina, o que subestima a importância dos casos. Estudos amplos e multicêntricos podem contribuir para a ampliação do conhecimento sobre os fatores de risco e sua prevenção para a população em geral. Além de auxiliar no diagnóstico para que possam ser tratados antes de apresentarem fatores irreversíveis para sua saúde e bem-estar.

De suma importância também frisar que, pela significante alteração de fatores predisponentes da SM em indivíduos tão jovens, a carga de patologias possivelmente será alta, impactando na qualidade de vida e na saúde coletiva de grande parcela da população mundial num futuro próximo.

\section{REFERÊNCIAS BIBLIOGRÁFICAS}

1. AZAMBUJA, C.R.; FARINHA, J.B; SANTOSA, D.L. O estilo de vida de mulheres com síndrome metabólica. Revista Baiana de Saúde Pública. V. 39, n. 2, p. 384-396, abr-jun, 2015.

2. BARBALHO, S.M.B. Comparação da prevalência de fatores de risco para a síndrome metabólica entre homens e mulheres acadêmicos de uma instituição pública de nível superior de Marília - SP. Revista Saúde \& Pesquisa. v. 2, n. 3, p. 345-348, set-dez, 2009.

3. BARBOSA, B.J. et al. Síndrome Metabólica em Ambulatório Cardiológico. Arquivos Brasileiros de Cardiologia, v. 94, n. 1, p. 44-55, 2010.

4. BARBOSA, J.B. et al. Metabolic syndrome, insulin resistance and other cardiovascular risk factors in university students. Ciência \& Saúde Coletiva. v. 4, n. 21, p. 1123-1136, 2016.

5. BRANDÃO, A.P. et al. I Diretriz Brasileira de Diagnóstico e Tratamento da Síndrome Metabólica. Arquivos Brasileiros de Cardiologia, v. 84, sup. 1, abr., 2005.

6. BORTOLETTO, M.S.S. et al. Síndrome Metabólica em Estudos com Adultos Brasileiros: Uma Revisão Sistemática. Revista Espaço para a Saúde, Londrina, v. 4, n. 15, p. 86-98, 2014.

7. CAMPOS, K.E. et al. Obesidade e Resistência à Insulina. Femina, v. 34, n. 9, 2006.

8. CAÑETE, R. et al. A Development of Insulin Resistance and Its Relation to Diet in the Obese Child. Revista Brasileira de Medicina do Esporte, São Paulo, v. 14, n. 3, p. 197-200, maio-jun, 2008. 
9. CAO, S. et al. J-shapedness: an often missed, often miscalculated relation: the example of weight and mortality. Jornal of Epidemiology and Public Health, Canada, n.68, p. 683-690, mar, 2014.

10. CARVALHO et al. Associação entre fatores de risco cardiovascular e indicadores antropométricos de obesidade em universitários de São Luís, Maranhão, Brasil. Ciência \& Saúde Coletiva. v. 2, n. 20, p. 479-490, 2015.

11. CASTANHO, G.K, et al. Consumo de frutas, verduras e legumes associado a Síndrome Metabólica e seus componentes em uma amostra populacional adulta. Ciência \& Saúde Coeltiva. V. 18, n. 2, p. 385-392, 2013.

12. CASTRO, S.H.; MATO H.J., GOMES, M.B. Parâmetros Antropométricos e Síndrome Metabólica em Diabetes Tipo 2. Arquivos Brasileiros de Endocrinologia e Metabologia, São Paulo, v. 50, n. 3, jun., 2006.

13. CESARETTI, M.L.R; KOHLMANNJR., O. Modelos Experimentais de Resistência à Insulina e Obesidade: Lições Aprendidas. Arquivo Brasileiro de Endocrinologia e Metabologia, v. 50, n. 2, p. 190-197, 2006.

14. CHOCAIR, P.R. Síndrome Metabólica - Conhecer para prevenir. http://sindromemetabolica.med.br/conceito-de-sobrepeso-e-obesidade/ acessado em 16/08/2017.

15. Consenso Brasileiro sobre Dislipidemias: Deteç̧ão, Avaliação e Tratamento. Sociedade Brasileira de Cardiologia 1994. Disponível em: http://publicacoes.cardiol.br/consenso/1994/6301/63010014.pdf. Acessado em: $17 / 08 / 2017$.

16. CRUZ, I.R.D. et al. Metabolic Syndrome and Its Associantion with Socioeconomic. Level in Students. Revista CEFAC. V. 16, n. 4, p. 1294-1301, jul-ago, 2014

17. DALLECK, L.C.; KIELLAND, E.M. The Prevalence of Metabolic Syndrome and Metabolic Syndrome Risk Factors in College-Aged Students. American Journal of Health Promotion. USA, v. 27, n. 1, p. 37-42, 2012.

18. DUTRA, E.S. Metabolic Syndrome in Central Brazil: Prevalence and Correlates in Adult Population. Diabetology and Metabolic Syndrome, v. 4, n. 20, 2012.

19. ECKEL, R.H.; GRUNDY, S.M.; ZIMMET, P.Z. The Metabolic Syndrome. Lancet, England, v. 365, n. 9468, p. 1415-1428, apr., 2005.

20. EICKHOFF, G.L.D., et al. Políticas públicas e a obesidade na adolescência. Disponível em: http://www.aninter.com.br/ANAIS\%201\%20CONITER/GT09\%20Sa\%FAde\%20e\% 20sociedade/POL\%CDTICAS\%20P\%DABLICAS\%20EA\%20OBESIDADE\%20N A\%20ADOLESC\%CANCIA\%20-\%20trablho\%20completo.pdf . Acessado em: 15 de agosto de 2017.

21. ESMAILLZADEH, A., et al. Fruit and vegetable intakes, C-reactive protein, and the metabolic syndrome. The American Journal of Clinical Nutrition. V.4, n.6, p.1489-1497, 2006.

22. FEITOSA, E.P.S.; DANTAS, C.A.O.; ANDRADE-WARTHA, E.R.S.; MARCELLINI, P.S.; MENDES-NETTO, R.S. Hábitos alimentares de estudantes de uma universidade pública no Nordeste, Brasil. Alimentos e Nutrição Araraquara. v.21, n.2, p.225-230, abril/junho, 2010.

23. FOCHAT, R.C. Qualidade da dieta de estudantes de uma universidade pública brasileira. Revista de APS. V.19, n.1, p: 95 - 105, janeiro/março, 2016. 
24. FERRARI, C.K.B. Atualização: Fisiopatologia e Clínica da Síndrome Metabólica. Arquivos Catarinenses de Medicina, v. 36, n. 4, p. 90-95, 2007.

25. FERREIRA, M.E. Síndrome metabólica e doenças cardiovasculares: do conceito ao tratamento. Arquivos Catarinenses de Medicina, v. 45, n. 4, p. 95-109, outubro-dezembro, 2016.

26. FEOLI, A.M.P.; GOTTLIEB, M.G. Intervenções Tecnológicas na Síndrome Metabólica: Novos Rumos para os Profissionais de Saúde. Scientia Medica. v. 49, n. 1, janeiro-fevvereiro, 2016.

27. GELONEZE, B.; TAMBASCIA, M.A. Avaliação Laboratorial e Diagnóstica da Resistência Insulínica. Arquivo Brasileiro de Endocrinologia e Metabologia, v. 50, n. 2, p. 208-215, abr., 2006.

28. GOODY-MATOS, A.F. Endocárdio Metabologia na Prática Clínica. Guanabara Koogan, São Paulo, p. 46, 2011.

29.GOTTLIEB, M.G.V.; CRUZ, I.B.M.; BODANESE, L.C. Origem da Síndrome Metabólica: Aspectos Evolutivos e Nutricionais. Sientia Médica, Porto Alegre, v. 18, n.1, p. 31-38, jan-mar, 2008.

30. HERMSDORFF, H.H.M.; MONTEIRO, J.B.R. Gordura Visceral, Subcutânea ou Intramuscular: Onde Está o Problema? Arquivo Brasileiro de Endocrinologia e Metabologia, v. 48, n. 6, dez., 2004.

31. INTERNATIONAL DIABETES FEDERATION. The IDF Consensus Worldwide Definition of the Metabolic Syndrome, IDF, Belgium, 2006.

32. KAUR, J. A comprehensive review on metabolic syndrome. Cardiology Research and Practice. Mac, 2014.

33. KUSCHNIR, M.C. et al. ERICA: Prevalence of Metabolic Syndrome in Brazilians Adolescents. Revista de Saúde Pública, v. 50, sup 1, 2016.

34. LAKKA, H.M. et al. The Metabolic Syndrome and Total and Cadiovascular Disease Mortality in Middle-aged Men. The Journal oh The American Medical Association, v. 428, n. 21, p. 2709-2716, dec., 2002.

35. LAM, D.W., LeROITH, D. Metabolic Syndrome. Endotext. Atualizada em 19/05/2015. Disponível em: https://www.ncbi.nlm.nih.gov/books/NBK278936/. Acessada em 19/08/2017.

36. LEÃO, L.S.C.S.; BARROS, E.G.; KOIFMAN R.J. Prevalência e Fatores Associados à Síndrome Metabólica em Usuários de Unidades Básicas de Saúde em São Paulo.Revista Brasileira de Cardiologia, v. 23, n. 2, mar-abr, 2010.

37. LUNA, R.L. Ponto de Vista: Síndrome Metabólica. Arquivo Brasileiro de Cardiologia, v.88, n. 5, p. 124-126, 2007.

38. LUTSEY, P.L et al. Dietary Intake and the Development of the Metabolic Syndrome: The Atherosclerosis Risk in Communities Study. Journal of the American Heart Association, jan, 2008.

39. MARCHI-ALVES, L.M. et al. Componentes da Síndrome Metabólica na Hipertensão Arterial. Revista da Escolade Enfermagem da USP, v. 46, n. 6, p. 1348-1353, 2012.

40.MANNA, T.D.; DAMIANI, D.; SETIAN, N. Síndrome Metabólica: Ensaios. Pediatria, São Paulo, v. 28, n. 4, p. 272-277, 2006.

41. MARTINI, F.A.N.; BORGES, M.B.; GUEDES, D.P. Hábito alimentar e síndrome metabólica em uma amostra de adultos brasileiros. Archivos Latinoamericanos de Nutrión. v.64, n.3, p.161-173, 2014. 
42. MORRELL, J.S.; COOK, S.B; CAREY, G.B. Cardiovascular Fitness, Activity and Metabolic Syndrome Among College Men and Women. Metabolic Syndrome and Related Disorders. USA, v. 11, n. 5, p. 370-376, 2013.

43. MORELL et al. Metabolic Syndrome: Comparison of Prevalence in Young Adults at 3 Land-Grant Universities. Journal of American College Health. USA, v. 62, jan, 2014.

44.OMS- Organização Mundial da Saúde. Disponível em: http://www.who.int/mediacentre/factsheets/fs312/en/. Acessado em 15 de agosto de 2017.

45. O'NEILL, S., O'DRISCOLL, L. Metabolic syndrome: a closer look at the growing epidemic and its associated pathologies. Obesity Reviews. v. 16, n. 1, p. 1-12, jan, 2015.

46. OTT, J.N. et al.Determinação da Prevalência de Síndrome Metabólica em Mulheres Pós-menopausa da Zona Rural de Catuíbe/RS. Revista Contexto \& Saúde. ljuí, v. 10, n. 20, jan-jun, 2011.

47.PAIXÃO, L.A.; DIAS, R.M.R.; PRADO, W.L. Estilo de vida e estado nutricional de universitários ingressantes em cursos da área de saúde do Recife/PE. Revista Brasileira de Atividade Física e Saúde. V.15, n.3, p.145-50, 2010.

48. PENALVA, D.Q.F. Síndrome Metabólica: Diagnóstico e Tratamento. Revista Médica, São Paulo, v. 87, n. 4, p. 245-250, out.-dez., 2008.

49. PINEDA, C.A. Síndrome Metabólico: Definición, Historia, Criterios. Enero-Marzo, v. 39, n. 1, 2008.

50.POZZAN, R. et al. Dislipidemia, Síndrome Metabólica e Risco Cardiovascular.Revista da Sociedade de Cardiologia do Rio de Janeiro, v. 17, n. 2, abr-jun, 2004.

51. RAMOS et al., 2013. Associação entre medidas antropométricas, de composição corporal, bioquímicas e de consumo alimentar em indivíduos com excesso de peso. Revista Brasileira de Promoção a Saúde, Fortaleza. V.26, n.2, p.157165, 2013.

52. REAVEN, G.M. Insulin resistance: from bit player to centre stage. Canadian Medical Association Journal. v.183, n. 5, mar, 2011.

53. REZENDE et al. Índice de Massa Corporal e Circunferência Abdominal: Associação com Fatores de Risco Cardiovascular. Arquivo Brasileiro de Cardiologia. V. 6, n. 87, p. 728-734, 2006.

54. RIBEIRO FILHO, F.F. et al. Gordura Visceral e Síndrome Metabólica: Mais do que uma Simples Associação. Arquivo Brasileiro de Endocrinologia e Metabologia, v. 2, n. 50, p. 230-238, 2006.

55. SALAROLI et al. Prevalência de Síndrome Metabólica em Estudo de Base Populacional, Vitória, ES - Brasil. Arquivo Brasileiro de Endocrinologiae Metabologia. v. 51, n. 7, p.1143-1152, 2007.

56. SILVA, A.R.V. et al. Prevalence of Metabolic Components in University Students, Revista Latino-Americana de Enfermagem. USP São Paulo, v. 6, n. 22, p. 1041-1047, 2014.

57. SILVA, C.C. et al. Circunferência do Pescoço como um Novo Indicador Antropométrico para Predição de Resistência à Insulina e Componentes da Síndrome Metabólica em Adolescentes: Brazilian Metabolic Syndrome Study.Revista Paulista de Pediatria, v. 32, n.2, p. 221-229, 2014. 
58. SILVA, D.A.D; SALVO, V.L.M.A. Prevalência de Síndrome Metabólica e Critérios Diagnósticos: Revisão. Revista Brasileira de Obesidade, Nutrição e Emagrecimento. São Paulo, v. 5, n. 28, jul-ago, 2011.

59. SINGH, N. et al. Prevalence of Metabolic Syndrome in Adolescents Aged 10-18 Years in Jammu, $\mathrm{J}$ and $\mathrm{K}$. Indian Journal of Endocrinology and Metabolism, v. 17, n. 1, p. 133-137, jan-feb, 2013.

60. Sociedade Brasileira de Diabetes, 2015. Síndrome metabólica em crianças e adolescentes. Disponível em: http://www.diabetes.org.br/profissionais/images/pdf/obesidadesindromemetabolica/001-Diretrizes-SBD-Sindrome-Metabolica-pg337.pdf Acessado em: 15 de agosto de 2017.

61. Third Report of the National Cholesterol Education Program (NCEP) Expert Panel on the Detection, Evaluation and Treatment of High Blood Cholesterol in Adults (Adult Treatment Panel III). Executive Sumary, MHI Publication 01, may 2001.

62. TURI, B.C. et al. Low Levels of Physical Activity and Metabolic Syndrome: Crosssectional Study I the Brazilian Health Sistem. Ciência e Saúde Coletiva, Rio de Janeiro, v. 21, n. 4, apr, 2016.

63. USHA, S.M.R.; CHANDRIKA, N.; SHETTY, R.R. A Study of the Components of Metabolic Syndrome in Young Adults. Biomedical Research. v. 25, n. 1, p. 4550, 2014.

64. VELÁZQUEZ, L.A.B.; HERRERA, C.J.A.C.; TAMAYOI, R.J. Factores de riesgo de síndrome metabólico en estudiantes de la universidad Pablo Guardado Chávez. Revista Cubana de Investigaciones Biomédicas. v. 32, n. 4, p. 379388, 2013.

65.WORLD HEALTH ORGANIZATION. Preventing Chronic Diseases: a Vital Investment. World Health Organization, WHO Global Report, Geneva, 2005. 


\section{APÊNDICE 1}

\section{QUESTIONÁRIO DE FREQÜÊNCIA ALIMENTAR ADULTO}

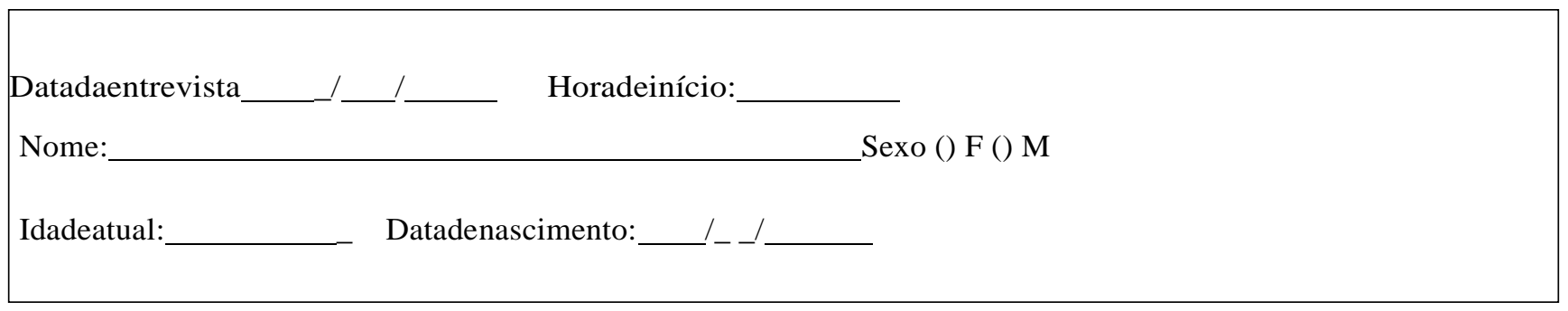

1.As questões seguintes relacionam-se ao seu hábito alimentar usual no PERÍODO DE UM ANO. Para cada quadro responda, por favor, a frequência que melhor descreva QUANTAS VEZES você costuma comer cada item e a respectiva UNIDADE DE TEMPO (se por dia, por semana, por mês ou no ano). Depois responda qual a sua PORÇÃO INDIVIDUAL USUAL em relação à porção média indicada. ESCOLHA SOMENTE UM CÍRCULO PARA CADA COLUNA. Muitos grupos de alimentos incluem exemplos. Eles são sugestões e você pode consumir todos os itens indicados. Se você não come ou raramente come um determinado item, preencha o círculodaprimeiracoluna( $\mathrm{N}=$ nuncacome).NÃODEIXEITENSEMBRANCO.

\begin{tabular}{|c|c|c|c|c|}
\hline \multirow{2}{*}{$\begin{array}{l}\text { GRUPO DE } \\
\text { ALIMENTOS }\end{array}$} & \multicolumn{2}{|c|}{ Com que frequência você costuma comer? } & \multicolumn{2}{|c|}{$\begin{array}{c}\text { Qualotamanhodesuaporçãoemrelaçãoà } \\
\text { porçãomédia? }\end{array}$} \\
\hline & QUANTAS VEZES VOCÊ COME: & UNIDADE & $\begin{array}{c}\text { PORÇÃO MÉDIA } \\
(\mathrm{M})\end{array}$ & SUA PORÇÃO \\
\hline $\begin{array}{l}\text { Alimentos e } \\
\text { preparações }\end{array}$ & $\begin{array}{l}\text { úmero de vezes: } 1,2,3 \text {, etc. } \\
(\mathrm{N}=\text { nunca ou raramente comeu } \\
\text { no último ano })\end{array}$ & $\begin{array}{l}\mathrm{D}=\text { por dia } \\
\mathrm{S}=\text { por semana } \\
\mathrm{M}=\text { por mês } \\
\mathrm{A}=\text { por ano }\end{array}$ & $\begin{array}{l}\text { Porção média de } \\
\text { referência }\end{array}$ & $\begin{array}{l}\mathrm{P}=\text { menor que a porção média } \\
\mathrm{M}=\text { igual à porção média } \\
\mathrm{G}=\text { maior que a porção média } \\
\mathrm{E}=\text { bem maior que a porção } \\
\text { média }\end{array}$ \\
\hline
\end{tabular}

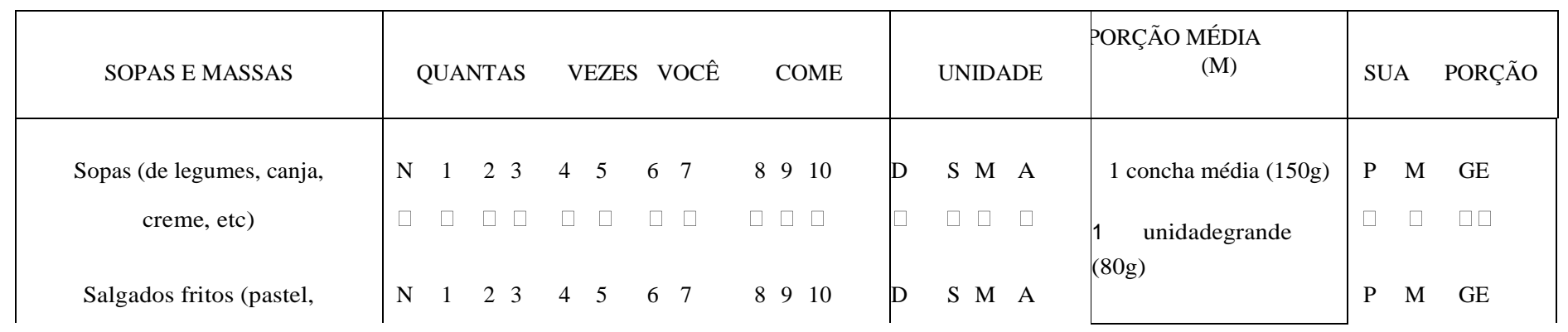




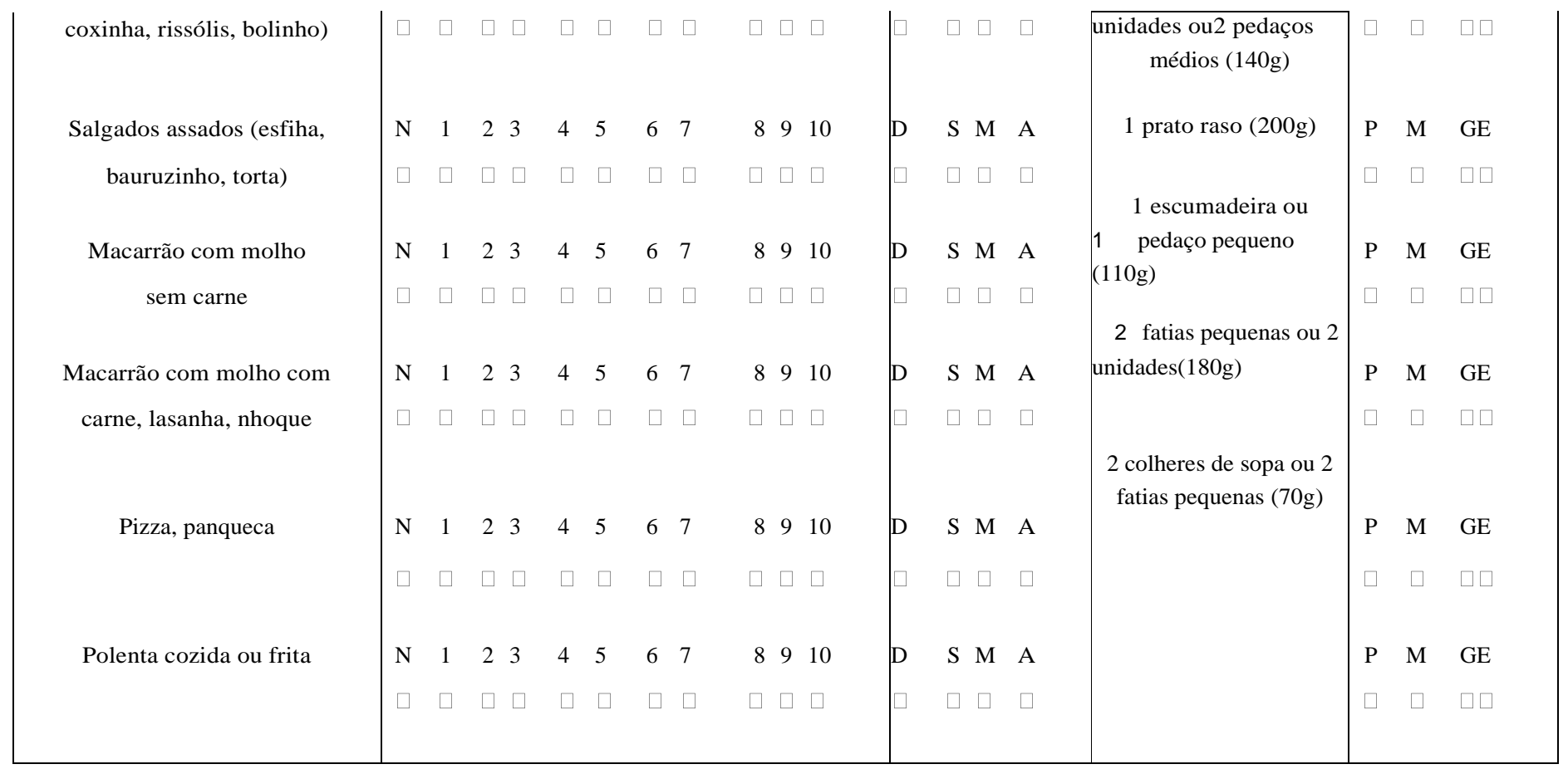




\begin{tabular}{|c|c|c|c|c|c|c|c|c|c|c|c|c|c|c|c|c|c|c|c|}
\hline \multirow[b]{2}{*}{$\begin{array}{l}\text { Carne de boi (bife, cozida, } \\
\text { assada), miúdos, vísceras }\end{array}$} & \multicolumn{4}{|c|}{ QUANTAS } & \multicolumn{3}{|c|}{ VEZES } & \multicolumn{2}{|c|}{ VOCÊ } & COME & \multicolumn{4}{|c|}{ UNIDADE } & \multirow{2}{*}{\begin{tabular}{|c}
$\begin{array}{c}\text { ORÇÃO MÉDIA } \\
(\mathrm{M})\end{array}$ \\
1 bife médio ou \\
2 pedaços $(100 \mathrm{~g})$
\end{tabular}} & \multicolumn{4}{|c|}{ SUA PORÇÃO } \\
\hline & $\begin{array}{l}\mathrm{N} \\
\square\end{array}$ & $\begin{array}{l}1 \\
\square\end{array}$ & $\begin{array}{l}2 \\
\square\end{array}$ & $\begin{array}{l}3 \\
\square\end{array}$ & $\begin{array}{l}4 \\
\square\end{array}$ & $\begin{array}{l}5 \\
\square\end{array}$ & $\begin{array}{l}6 \\
\square\end{array}$ & $\begin{array}{l}7 \\
\square\end{array}$ & $\begin{array}{l}8 \\
\square\end{array}$ & $\begin{array}{l}9 \quad 10 \\
\square \quad \square\end{array}$ & $\mathrm{D}$ & $\begin{array}{l}S \\
\square\end{array}$ & $\begin{array}{l}\mathrm{M} \\
\square\end{array}$ & $\begin{array}{l}\mathrm{A} \\
\square\end{array}$ & & $\begin{array}{l}\mathrm{P} \\
\square\end{array}$ & $\begin{array}{l}\mathrm{M} \\
\square\end{array}$ & $\begin{array}{l}\mathrm{G} \\
\square\end{array}$ & $\begin{array}{l}\mathrm{E} \\
\square\end{array}$ \\
\hline $\begin{array}{c}\text { Carne de porco (lombo, } \\
\text { bisteca) }\end{array}$ & $\begin{array}{l}\mathrm{N} \\
\square\end{array}$ & $\begin{array}{l}1 \\
\square\end{array}$ & $\begin{array}{l}2 \\
\square\end{array}$ & $\begin{array}{l}3 \\
\square\end{array}$ & $\begin{array}{l}4 \\
\square\end{array}$ & $\begin{array}{l}5 \\
\square\end{array}$ & $\begin{array}{l}6 \\
\square\end{array}$ & $\begin{array}{l}7 \\
\square\end{array}$ & $\begin{array}{l}8 \\
\square\end{array}$ & $\begin{array}{l}9 \quad 10 \\
\square \quad \square\end{array}$ & D & $\begin{array}{l}S \\
\square\end{array}$ & $\begin{array}{l}\mathrm{M} \\
\square\end{array}$ & $\begin{array}{l}\mathrm{A} \\
\square\end{array}$ & 1 fatia média $(100 \mathrm{~g})$ & $\begin{array}{l}\mathrm{P} \\
\square\end{array}$ & $\begin{array}{l}\mathrm{M} \\
\square\end{array}$ & $\begin{array}{l}\mathrm{G} \\
\square\end{array}$ & $\begin{array}{l}\mathrm{E} \\
\square\end{array}$ \\
\hline $\begin{array}{l}\text { Carne seca, carne de sol, } \\
\text { bacon }\end{array}$ & $\begin{array}{l}\mathrm{N} \\
\square\end{array}$ & $\begin{array}{l}1 \\
\square\end{array}$ & $\begin{array}{l}2 \\
\square\end{array}$ & $\begin{array}{l}3 \\
\square\end{array}$ & $\begin{array}{l}4 \\
\square\end{array}$ & $\begin{array}{l}5 \\
\square\end{array}$ & $\begin{array}{l}6 \\
\square\end{array}$ & $\begin{array}{l}7 \\
\square\end{array}$ & $\begin{array}{l}8 \\
\square\end{array}$ & $\begin{array}{l}9 \quad 10 \\
\square \quad \square\end{array}$ & $\mathrm{D}$ & $\begin{array}{l}S \\
\square\end{array}$ & $\begin{array}{c}M \\
\square\end{array}$ & $\begin{array}{c}\mathrm{A} \\
\square\end{array}$ & $\begin{array}{l}2 \text { pedaços pequenos } \\
(40 \mathrm{~g})\end{array}$ & $\begin{array}{l}\mathrm{P} \\
\square\end{array}$ & $\begin{array}{c}M \\
\square\end{array}$ & $\begin{array}{l}\mathrm{G} \\
\square\end{array}$ & $\begin{array}{l}\mathrm{E} \\
\square\end{array}$ \\
\hline Linguiça & $\begin{array}{l}\mathrm{N} \\
\square\end{array}$ & $\begin{array}{l}1 \\
\square\end{array}$ & $\begin{array}{l}2 \\
\square\end{array}$ & $\begin{array}{l}3 \\
\square\end{array}$ & $\begin{array}{l}4 \\
\square\end{array}$ & $\begin{array}{l}5 \\
\square\end{array}$ & $\begin{array}{l}6 \\
\square\end{array}$ & $\begin{array}{l}7 \\
\square\end{array}$ & $\begin{array}{l}8 \\
\square\end{array}$ & $\begin{array}{l}9 \quad 10 \\
\square \quad \square\end{array}$ & $\mathrm{D}$ & $\begin{array}{l}S \\
\square\end{array}$ & $\begin{array}{c}M \\
\square\end{array}$ & & 1 gomo médio $(60 \mathrm{~g})$ & $\begin{array}{l}\mathrm{P} \\
\square\end{array}$ & $\begin{array}{c}M \\
\square\end{array}$ & $\begin{array}{l}\mathrm{G} \\
\square\end{array}$ & $\begin{array}{c}\mathrm{E} \\
\square\end{array}$ \\
\hline $\begin{array}{c}\text { Embutidos (presunto, } \\
\text { mortadela, salsicha) }\end{array}$ & $\begin{array}{l}\mathrm{N} \\
\square\end{array}$ & $\begin{array}{l}1 \\
\square\end{array}$ & $\begin{array}{l}2 \\
\square\end{array}$ & $\begin{array}{l}3 \\
\square\end{array}$ & $\begin{array}{l}4 \\
\square\end{array}$ & $\begin{array}{l}5 \\
\square\end{array}$ & $\begin{array}{l}6 \\
\square\end{array}$ & $\begin{array}{l}7 \\
\square\end{array}$ & $\begin{array}{l}8 \\
\square\end{array}$ & $\begin{array}{l}9 \quad 10 \\
\square \quad \square\end{array}$ & $\mathrm{D}$ & $\begin{array}{l}S \\
\square\end{array}$ & $\begin{array}{c}M \\
\square\end{array}$ & $\begin{array}{l}\mathrm{A} \\
\square\end{array}$ & 2 fatias médias $(30 \mathrm{~g})$ & $\begin{array}{l}\mathrm{P} \\
\square\end{array}$ & $\begin{array}{c}M \\
\square\end{array}$ & $\begin{array}{l}\mathrm{G} \\
\square\end{array}$ & $\begin{array}{l}\mathrm{E} \\
\square\end{array}$ \\
\hline $\begin{array}{l}\text { Frango (cozido, frito, } \\
\text { grelhado, assado) }\end{array}$ & $\begin{array}{l}\mathrm{N} \\
\square\end{array}$ & $\begin{array}{l}1 \\
\square\end{array}$ & $\begin{array}{l}2 \\
\square\end{array}$ & $\begin{array}{l}3 \\
\square\end{array}$ & $\begin{array}{l}4 \\
\square\end{array}$ & $\begin{array}{l}5 \\
\square\end{array}$ & $\begin{array}{l}6 \\
\square\end{array}$ & $\begin{array}{l}7 \\
\square\end{array}$ & $\square$ & $\begin{array}{l}9 \quad 10 \\
\square \quad \square\end{array}$ & $\mathrm{D}$ & $\begin{array}{l}S \\
\square\end{array}$ & $\begin{array}{l}\mathrm{M} \\
\square\end{array}$ & $\begin{array}{l}\mathrm{A} \\
\square\end{array}$ & $\begin{array}{l}1 \text { pedaço ou } 1 \text { filé } \\
\text { pequeno }(60 \mathrm{~g})\end{array}$ & $\begin{array}{l}\mathrm{P} \\
\square\end{array}$ & $\begin{array}{l}\mathrm{M} \\
\square\end{array}$ & $\begin{array}{l}\mathrm{G} \\
\square\end{array}$ & $\begin{array}{l}\mathrm{E} \\
\square\end{array}$ \\
\hline $\begin{array}{c}\text { Hambúrguer, nuggets, } \\
\text { almôndega }\end{array}$ & $\begin{array}{l}\mathrm{N} \\
\square\end{array}$ & $\begin{array}{l}1 \\
\square\end{array}$ & $\begin{array}{l}2 \\
\square\end{array}$ & $\begin{array}{l}3 \\
\square\end{array}$ & $\begin{array}{l}4 \\
\square\end{array}$ & $\begin{array}{l}5 \\
\square\end{array}$ & $\begin{array}{l}6 \\
\square\end{array}$ & $\begin{array}{l}7 \\
\square\end{array}$ & $\begin{array}{l}8 \\
\square\end{array}$ & $\begin{array}{l}9 \quad 10 \\
\square \quad \square\end{array}$ & $\mathrm{D}$ & $\begin{array}{l}S \\
\square\end{array}$ & $\begin{array}{l}\mathrm{M} \\
\square\end{array}$ & $\begin{array}{l}\mathrm{A} \\
\square\end{array}$ & 1 unidade média $(60 \mathrm{~g})$ & $\square$ & $\begin{array}{c}\mathrm{M} \\
\square\end{array}$ & $\begin{array}{l}\mathrm{G} \\
\square\end{array}$ & $\begin{array}{l}\mathrm{E} \\
\square\end{array}$ \\
\hline $\begin{array}{l}\text { Peixe (cozido, frito, } \\
\text { assado) e frutos do mar }\end{array}$ & $\begin{array}{l}\mathrm{N} \\
\square\end{array}$ & $\begin{array}{l}1 \\
\square\end{array}$ & $\begin{array}{l}2 \\
\square\end{array}$ & $\begin{array}{l}3 \\
\square\end{array}$ & $\begin{array}{l}4 \\
\square\end{array}$ & $\begin{array}{l}5 \\
\square\end{array}$ & $\begin{array}{l}6 \\
\square\end{array}$ & $\begin{array}{l}7 \\
\square\end{array}$ & $\begin{array}{l}8 \\
\square\end{array}$ & $\begin{array}{l}9 \quad 10 \\
\square \quad \square\end{array}$ & $\mathrm{D}$ & $\begin{array}{l}S \\
\square\end{array}$ & $\begin{array}{c}M \\
\square\end{array}$ & $\begin{array}{l}\mathrm{A} \\
\square\end{array}$ & $\begin{array}{l}1 \text { filé pequeno ou } \\
1 \text { posta pequena } \\
(100 \mathrm{~g})\end{array}$ & $\square$ & $\begin{array}{c}\mathrm{M} \\
\square\end{array}$ & $\begin{array}{l}\mathrm{G} \\
\square\end{array}$ & \\
\hline
\end{tabular}

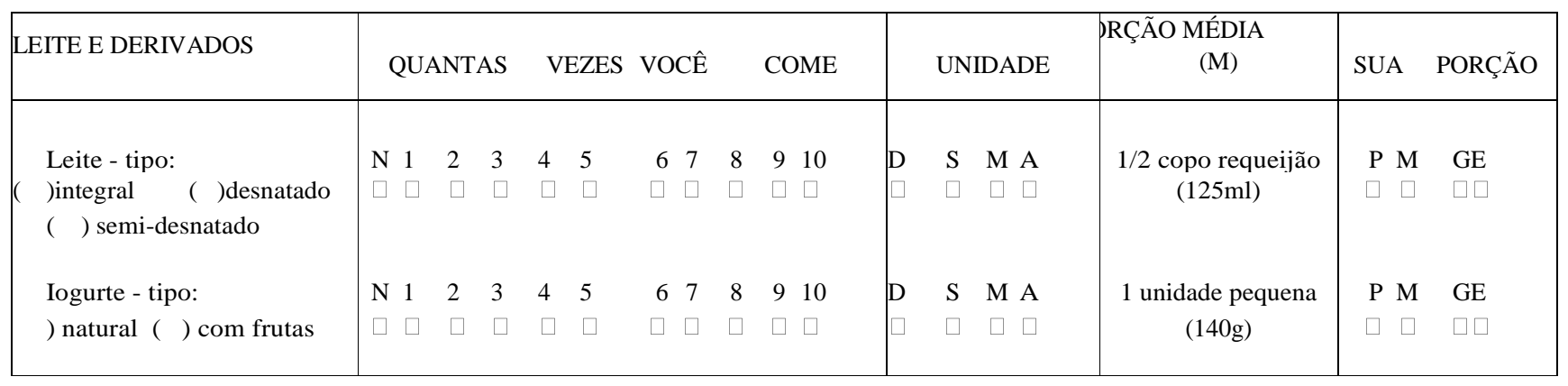

\begin{tabular}{|c|c|c|c|c|c|c|c|c|c|c|c|c|c|c|c|}
\hline \multirow{2}{*}{$\begin{array}{l}\text { LEITE E DERIVADOS } \\
\text { Queijo mussarela, prato, }\end{array}$} & \multicolumn{3}{|c|}{ QUANTAS } & \multicolumn{2}{|c|}{ VEZES } & \multicolumn{3}{|c|}{ VOCÊ } & COME & \multicolumn{3}{|c|}{ UNIDADE } & $\begin{array}{c}\text { RŖÃO MÉDIA } \\
\text { (M) }\end{array}$ & SUA & PORÇÃC \\
\hline & N 1 & 2 & 3 & 4 & 5 & 6 & 7 & 8 & $9 \quad 10$ & D & $S$ & $\mathrm{M} \mathrm{A}$ & $11 / 2$ fatias grossas & $\mathrm{P}$ & GE \\
\hline parmesão, provolone & $\square \square$ & $\square$ & $\square$ & $\square$ & $\square$ & $\square$ & $\square$ & $\square$ & $\square \square$ & $\square$ & $\square$ & $\square \square$ & $(30 g)$ & $\square$ & $\square \square$ \\
\hline \multirow[t]{2}{*}{ Queijo minas, ricotta } & N 1 & 2 & 3 & 4 & 5 & & 7 & 8 & $9 \quad 10$ & D & S & M A & 1 fatia média $(30 \mathrm{~g})$ & $\mathrm{P}$ & GE \\
\hline & $\square \square$ & $\square$ & $\square$ & $\square$ & $\square$ & $\square$ & $\square$ & $\square$ & $\square \square$ & $\square$ & & $\square \square$ & & $\square$ & म० \\
\hline
\end{tabular}




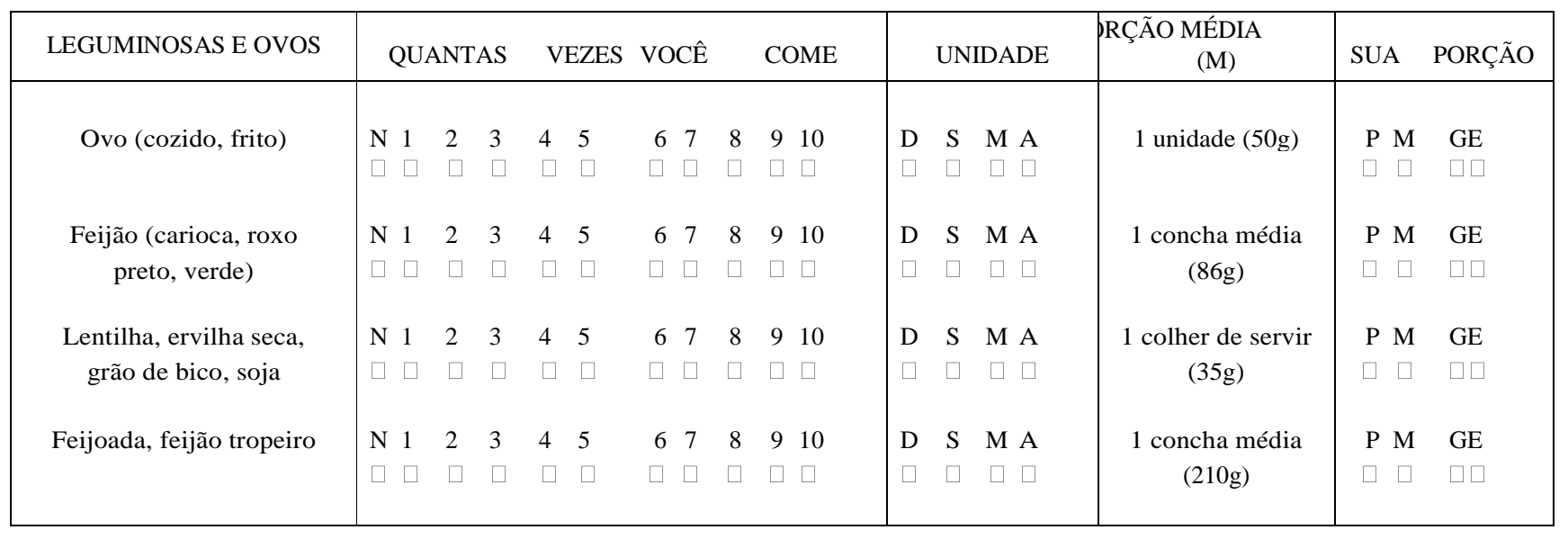

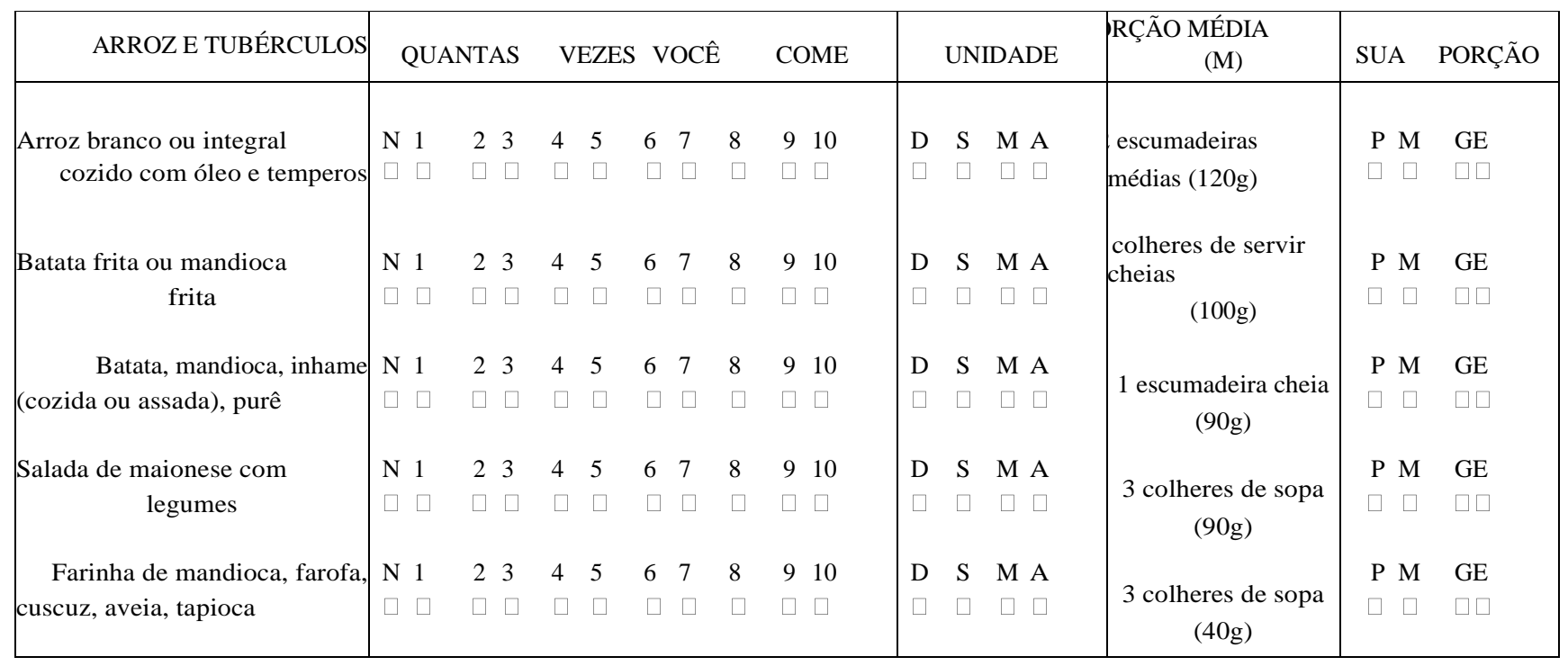

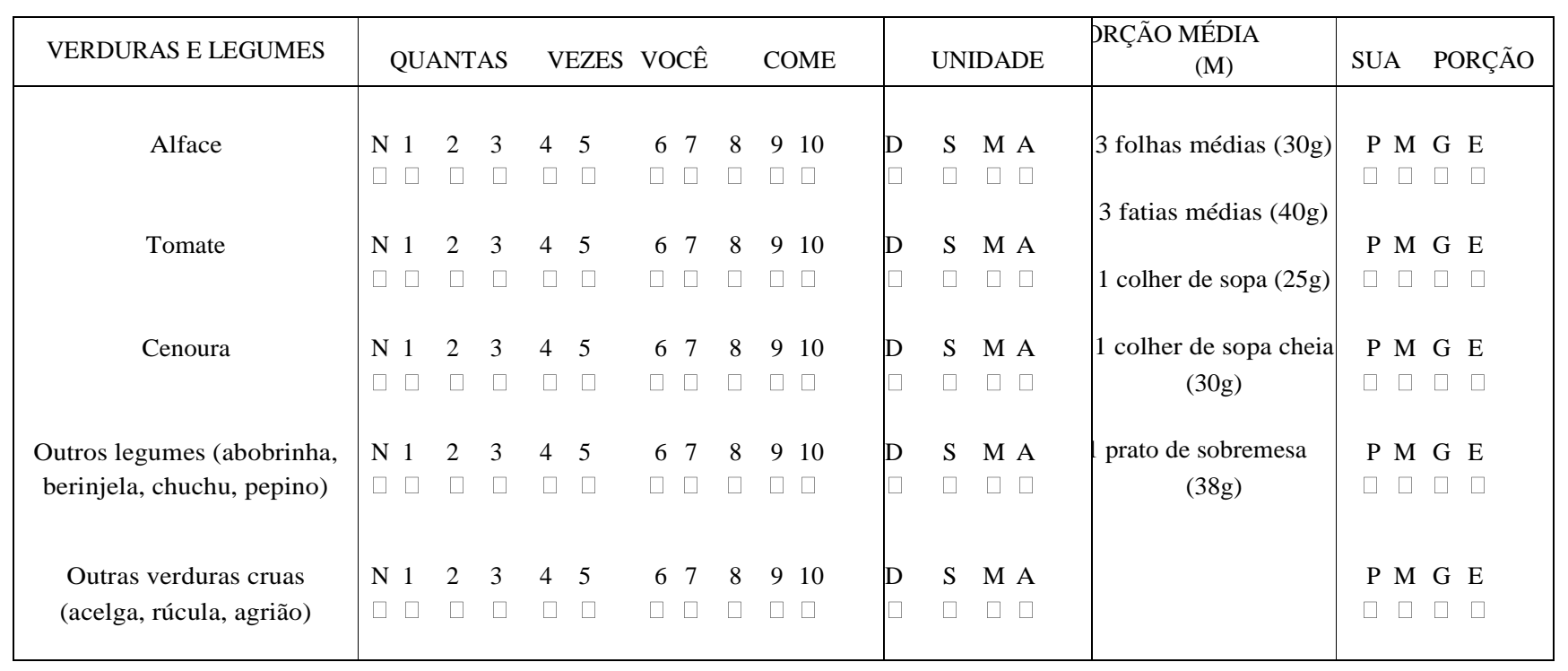




\begin{tabular}{|c|c|c|c|c|c|c|c|c|c|c|c|c|c|c|c|c|c|c|}
\hline VERDURAS E LEGUMES & & QUA & NTAS & & EZES & & OCÊ & & COME & & & JIDA & $\mathrm{DE}$ & $\begin{array}{c}\text { PRÇÃO MÉDIA } \\
(\mathrm{M})\end{array}$ & & UA & & ORÇÃO \\
\hline $\begin{array}{l}\text { Outras verduras cozidas } \\
\text { (acelga, espinafre, escarola, }\end{array}$ & $\mathrm{N}$ & 1 & 23 & 4 & 5 & 6 & 7 & 8 & $\begin{array}{ll}9 & 10\end{array}$ & D & $\mathrm{S}$ & M & A & 1 colher deservir & $P$ & M & G & E \\
\hline couve) & $\square$ & $\square$ & $\square \sqsupset$ & $\sqsupset$ & $\sqsupset$ & $\sqsupset$ & $\sqsupset$ & $\sqsupset$ & $\square \square$ & 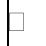 & $\sqsupset$ & $\square$ & $\square$ & $(30 g)$ & ] & $\square$ & $\square$ & $\sqsupset$ \\
\hline Brócolis, couve-flor, repolho & $\begin{array}{l}N \\
\square\end{array}$ & $\begin{array}{l}1 \\
\square\end{array}$ & $\begin{array}{l}23 \\
\square \quad \square\end{array}$ & $\begin{array}{l}4 \\
\square\end{array}$ & $\begin{array}{l}5 \\
\square\end{array}$ & $\begin{array}{l}6 \\
\square\end{array}$ & $\begin{array}{l}7 \\
\square\end{array}$ & $\begin{array}{l}8 \\
\square\end{array}$ & $\begin{array}{l}9 \quad 10 \\
\square \quad \square\end{array}$ & $\mathrm{D}$ & $\begin{array}{l}\mathrm{S} \\
\square\end{array}$ & $\begin{array}{c}\mathrm{M} \\
\square\end{array}$ & $\begin{array}{l}\text { A } \\
\square\end{array}$ & $\begin{array}{l}\quad 1 \text { ramo ou } \\
2 \text { colheres desopa } \\
(30 \mathrm{~g})\end{array}$ & $\mathrm{P}$ & $\begin{array}{l}M \\
\square\end{array}$ & $\begin{array}{l}\mathrm{G} \\
\square\end{array}$ & $\begin{array}{l}\mathrm{E} \\
\square\end{array}$ \\
\hline
\end{tabular}

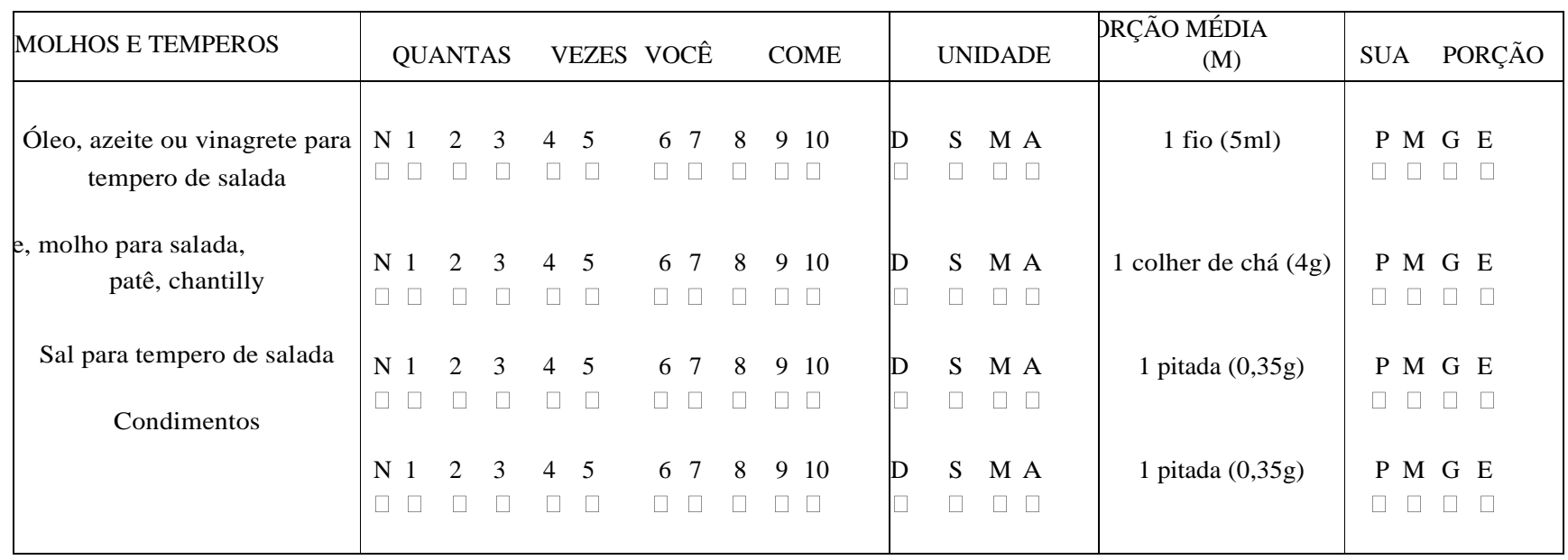

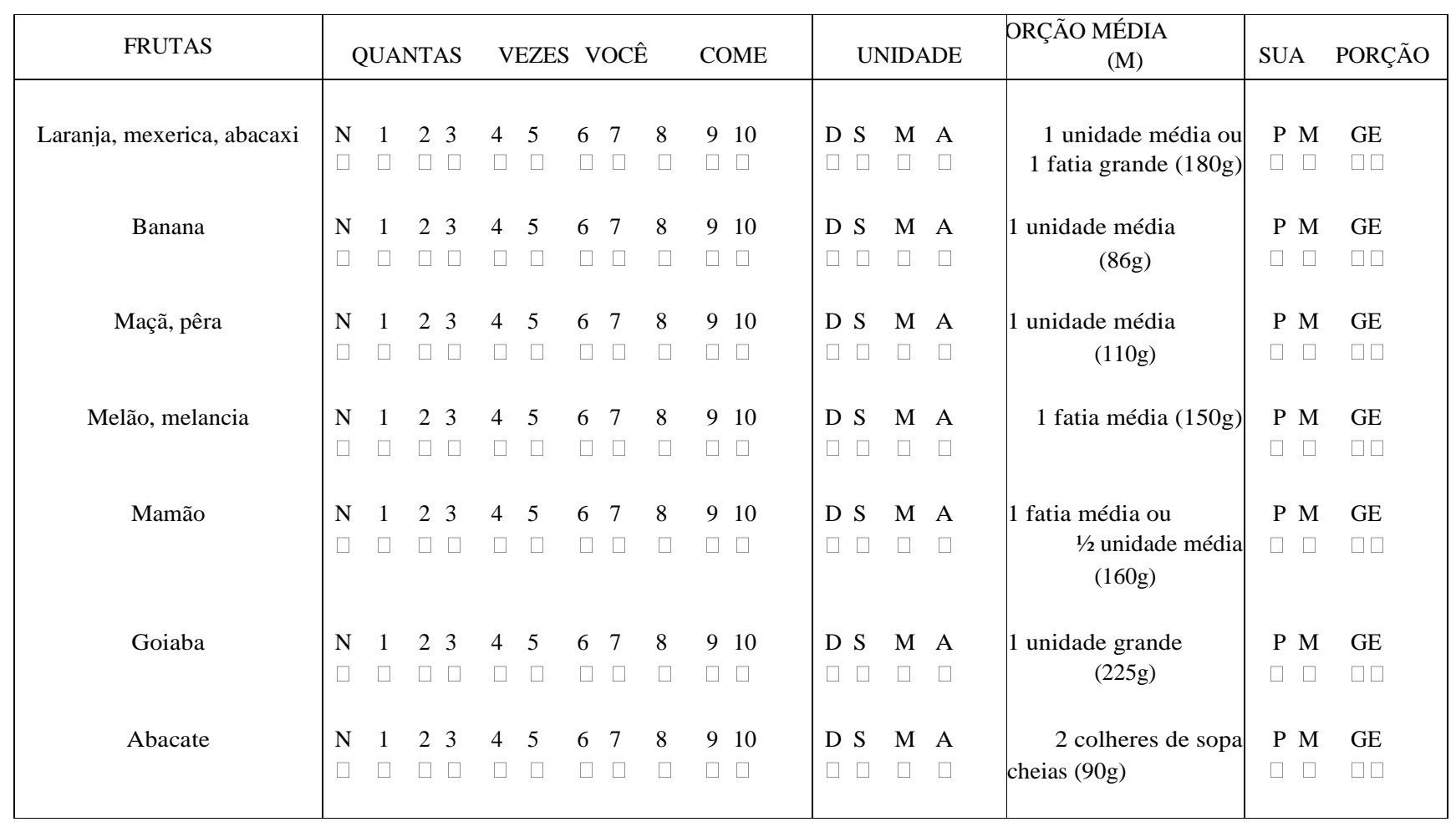




\begin{tabular}{|c|c|c|c|c|c|c|c|c|c|c|c|c|c|c|c|c|}
\hline BEBIDAS & \multicolumn{3}{|c|}{ QUANTAS } & \multicolumn{2}{|c|}{ VEZES } & \multicolumn{3}{|c|}{ VOCÊ } & COME & \multicolumn{4}{|c|}{ UNIDADE } & $\begin{array}{c}\text { ORÇÃO MÉDIA } \\
(\mathrm{M})\end{array}$ & SUA & PORÇÃO \\
\hline Suco natural & $\begin{array}{l}\mathrm{N} \\
\square\end{array}$ & $\begin{array}{l}1 \\
\square\end{array}$ & $\begin{array}{l}23 \\
\square \square\end{array}$ & $\begin{array}{l}4 \\
\square\end{array}$ & $\begin{array}{l}5 \\
\square\end{array}$ & $\begin{array}{l}6 \\
\square\end{array}$ & $\begin{array}{l}7 \\
\square\end{array}$ & $\begin{array}{l}8 \\
\square\end{array}$ & $\begin{array}{l}9 \quad 10 \\
\square \quad \square\end{array}$ & $\begin{array}{l}\mathrm{D} \\
\square\end{array}$ & $\begin{array}{l}S \\
\square\end{array}$ & $\begin{array}{l}M \\
\square\end{array}$ & $\begin{array}{l}\mathrm{A} \\
\square\end{array}$ & $\begin{array}{c}1 / 2 \text { copo americano } \\
(80 \mathrm{ml})\end{array}$ & & $\begin{array}{l}\mathrm{GE} \\
\square \square\end{array}$ \\
\hline Suco industrializado & $\begin{array}{l}\mathrm{N} \\
\square\end{array}$ & $\begin{array}{l}1 \\
\square\end{array}$ & $\begin{array}{l}23 \\
\square \square\end{array}$ & $\begin{array}{l}4 \\
\square\end{array}$ & $\begin{array}{l}5 \\
\square\end{array}$ & $\begin{array}{l}6 \\
\square\end{array}$ & $\begin{array}{l}7 \\
\square\end{array}$ & $\begin{array}{l}8 \\
\square\end{array}$ & $\begin{array}{l}9 \quad 10 \\
\square \quad \square\end{array}$ & $\mathrm{D}$ & $\begin{array}{l}S \\
\square\end{array}$ & $\begin{array}{l}M \\
\square\end{array}$ & $\begin{array}{l}\text { A } \\
\square\end{array}$ & $\begin{array}{c}1 \text { copo de requeijão } \\
(240 \mathrm{ml})\end{array}$ & $\begin{array}{l}\mathrm{P} \Lambda \\
\square \square\end{array}$ & $\begin{array}{l}\text { GE } \\
\square \square\end{array}$ \\
\hline Café ou chá sem açúcar & $\mathrm{N}$ & 1 & 23 & 4 & 5 & 6 & 7 & 8 & $9 \quad 10$ & $\mathrm{D}$ & $\mathrm{S}$ & M & A & 2 xícaras de café & $\mathrm{P} N$ & GE \\
\hline
\end{tabular}

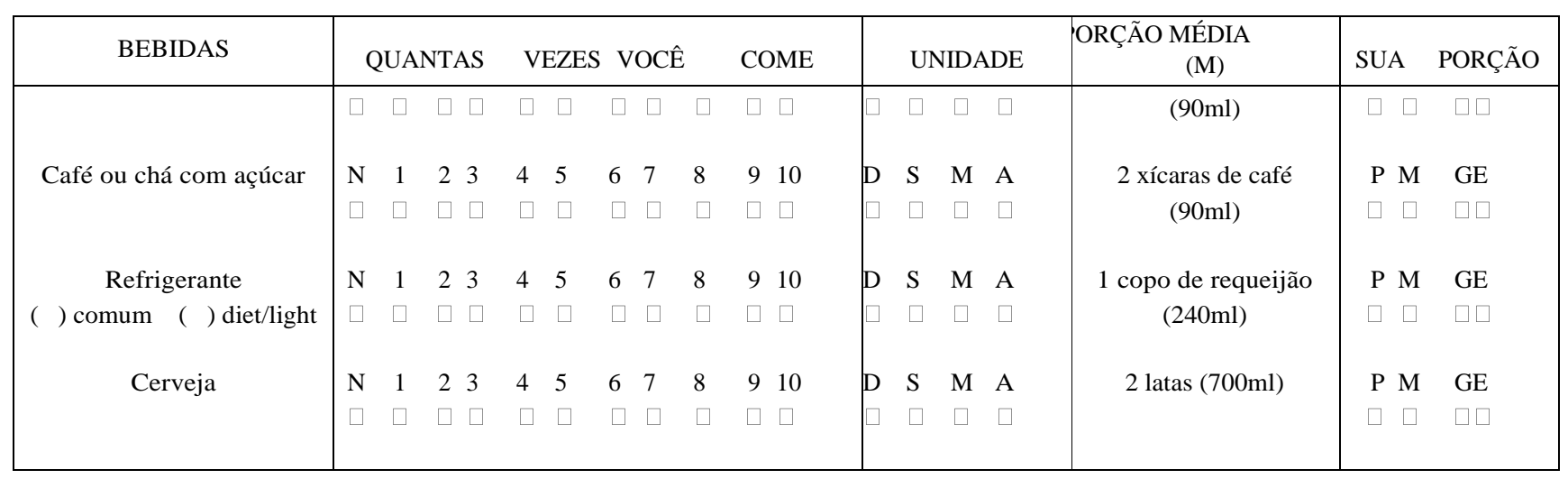




\begin{tabular}{|c|c|c|c|c|c|c|c|c|c|c|c|c|c|c|c|c|c|}
\hline PÃES E BISCOITOS & \multicolumn{3}{|c|}{ QUANTAS } & \multicolumn{2}{|c|}{ VEZES } & \multicolumn{3}{|c|}{ VOCÊ } & COME & \multicolumn{3}{|c|}{ UNIDADE } & \multicolumn{2}{|r|}{$\begin{array}{c}\text { ORÇÃO MÉDIA } \\
(\mathrm{M})\end{array}$} & \multicolumn{3}{|c|}{ SUA } \\
\hline $\begin{array}{l}\text { Pão francês, pão de forma, } \\
\text { integral, pão doce, torrada }\end{array}$ & $\begin{array}{l}\text { N } 1 \\
\square \quad \square\end{array}$ & $\begin{array}{l}2 \\
\square\end{array}$ & $\begin{array}{l}3 \\
\square\end{array}$ & $\begin{array}{l}4 \\
\square\end{array}$ & $\begin{array}{l}5 \\
\square\end{array}$ & $\begin{array}{l}6 \\
\square\end{array}$ & $\begin{array}{l}7 \\
\square\end{array}$ & $\begin{array}{l}8 \\
\square\end{array}$ & $\begin{array}{ll}9 & 10 \\
\square & \square\end{array}$ & $\begin{array}{l}\mathrm{D} \\
\square\end{array}$ & $\begin{array}{l}S \\
\square\end{array}$ & $\begin{array}{l}\mathrm{M} \\
\square\end{array}$ & $\begin{array}{l}\mathrm{A} \\
\square\end{array}$ & $\begin{array}{l}1 \text { unidade ou } 2 \\
\text { fatias }(50 \mathrm{~g})\end{array}$ & $\begin{array}{l}\text { P M } \\
\square \square\end{array}$ & $\begin{array}{l}\mathrm{G} \\
\square\end{array}$ & $\begin{array}{l}\mathrm{E} \\
\square\end{array}$ \\
\hline $\begin{array}{l}\text { Biscoito sem recheio } \\
\quad \text { (doce, salgado) }\end{array}$ & $\begin{array}{l}\text { N } 1 \\
\square \quad \square\end{array}$ & $\begin{array}{l}2 \\
\square\end{array}$ & $\begin{array}{l}3 \\
\square\end{array}$ & $\begin{array}{l}4 \\
\square\end{array}$ & $\begin{array}{l}5 \\
\square\end{array}$ & $\begin{array}{c}6 \\
\square\end{array}$ & $\begin{array}{l}7 \\
\square\end{array}$ & $\begin{array}{l}8 \\
\square\end{array}$ & $\begin{array}{l}910 \\
\square \quad \square\end{array}$ & $\begin{array}{l}\mathrm{D} \\
\square\end{array}$ & $\begin{array}{l}S \\
\square\end{array}$ & $\begin{array}{c}\mathrm{M} \\
\square\end{array}$ & $\begin{array}{l}\mathrm{A} 4 \\
\square\end{array}$ & 4 unidades $(24 \mathrm{~g})$ & $\begin{array}{l}\text { P M } \\
\square \square\end{array}$ & $\begin{array}{l}\mathrm{G} \\
\square\end{array}$ & $\begin{array}{c}\mathrm{E} \\
\square\end{array}$ \\
\hline $\begin{array}{c}\text { Biscoito recheado, waffer, } \\
\text { amanteigado }\end{array}$ & $\begin{array}{l}\text { N } 1 \\
\square \quad \square\end{array}$ & $\begin{array}{l}2 \\
\square\end{array}$ & $\begin{array}{l}3 \\
\square\end{array}$ & $\begin{array}{l}4 \\
\square\end{array}$ & $\begin{array}{l}5 \\
\square\end{array}$ & $\begin{array}{c}6 \\
\square\end{array}$ & $\begin{array}{l}7 \\
\square\end{array}$ & 8 & $\begin{array}{l}910 \\
\square \quad \square\end{array}$ & $\begin{array}{l}\mathrm{D} \\
\square\end{array}$ & $\begin{array}{l}S \\
\square\end{array}$ & $\begin{array}{c}\mathrm{M} \\
\square\end{array}$ & $\begin{array}{l}\mathrm{A} \\
\square\end{array}$ & 3 unidades $(41 \mathrm{~g})$ & $\begin{array}{l}\text { P M } \\
\square \quad \square\end{array}$ & $\begin{array}{l}\mathrm{G} \\
\square\end{array}$ & $\begin{array}{l}\mathrm{E} \\
\square\end{array}$ \\
\hline Bolo (simples, recheado) & $\begin{array}{l}\text { N } 1 \\
\square \quad \square\end{array}$ & $\begin{array}{l}2 \\
\square\end{array}$ & $\begin{array}{l}3 \\
\square\end{array}$ & $\begin{array}{l}4 \\
\square\end{array}$ & $\begin{array}{l}5 \\
\square\end{array}$ & $\begin{array}{c}6 \\
\square\end{array}$ & $\begin{array}{l}7 \\
\square\end{array}$ & $\begin{array}{l}8 \\
\square\end{array}$ & $\begin{array}{l}9 \quad 10 \\
\square \quad \square\end{array}$ & $\begin{array}{l}\mathrm{D} \\
\square\end{array}$ & $\begin{array}{l}\mathrm{S} \\
\square\end{array}$ & $\begin{array}{c}\mathrm{M} \\
\square\end{array}$ & $\begin{array}{l}\mathrm{A} \\
\square\end{array}$ & 1 fatia média $(60 \mathrm{~g})$ & $\begin{array}{l}\text { P M } \\
\square \square\end{array}$ & $\begin{array}{l}\mathrm{G} \\
\square\end{array}$ & $\begin{array}{c}\mathrm{E} \\
\square\end{array}$ \\
\hline $\begin{array}{l}\text { Manteiga ou margarina } \\
\text { passada no pão } \\
\text { ( )comum } \quad \text { ( )light }\end{array}$ & $\begin{array}{l}\text { N } 1 \\
\square \quad \square\end{array}$ & $\begin{array}{l}2 \\
\square\end{array}$ & $\begin{array}{l}3 \\
\square\end{array}$ & $\begin{array}{l}4 \\
\square\end{array}$ & $\begin{array}{l}5 \\
\square\end{array}$ & $\begin{array}{l}6 \\
\square\end{array}$ & $\begin{array}{l}7 \\
\square\end{array}$ & $\begin{array}{l}8 \\
\square\end{array}$ & $\begin{array}{l}910 \\
\square \quad \square\end{array}$ & $\begin{array}{l}\mathrm{D} \\
\square\end{array}$ & $\begin{array}{l}S \\
\square\end{array}$ & $\begin{array}{c}\text { MA } \\
\square \square\end{array}$ & & $\begin{array}{c}3 \text { pontas de faca } \\
(15 \mathrm{~g})\end{array}$ & $\begin{array}{l}\text { P M } \\
\square \square\end{array}$ & $\begin{array}{l}\mathrm{G} \\
\square\end{array}$ & $\begin{array}{c}\mathrm{E} \\
\square\end{array}$ \\
\hline $\begin{array}{c}\text { Sanduíche (cachorro-quente, } \\
\text { hambúrguer) }\end{array}$ & $\begin{array}{l}\text { N } 1 \\
\square \quad \square\end{array}$ & $\begin{array}{l}2 \\
\square\end{array}$ & $\begin{array}{l}3 \\
\square\end{array}$ & $\begin{array}{l}4 \\
\square\end{array}$ & $\begin{array}{l}5 \\
\square\end{array}$ & $\begin{array}{l}6 \\
\square\end{array}$ & $\begin{array}{l}7 \\
\square\end{array}$ & $\begin{array}{l}8 \\
\square\end{array}$ & $\begin{array}{l}9 \quad 10 \\
\square \quad \square\end{array}$ & $\begin{array}{l}\mathrm{D} \\
\square\end{array}$ & $\begin{array}{l}S \\
\square\end{array}$ & $\begin{array}{c}\text { MA } \\
\square \square\end{array}$ & & $\begin{array}{l}2 \text { unidades simples } \\
(220 \mathrm{~g})\end{array}$ & $\begin{array}{l}\text { P M } \\
\square \square\end{array}$ & $\begin{array}{l}\mathrm{G} \\
\square\end{array}$ & $\begin{array}{c}\mathrm{E} \\
\square\end{array}$ \\
\hline
\end{tabular}

\begin{tabular}{|c|c|c|c|c|c|c|c|c|c|c|c|c|c|c|c|}
\hline DOCES E SOBREMESAS & \multicolumn{3}{|c|}{ QUANTAS } & \multicolumn{2}{|c|}{ VEZES } & \multicolumn{3}{|c|}{ VOCÊ } & COME & \multicolumn{3}{|c|}{ UNIDADE } & \multirow{2}{*}{$\begin{array}{c}\text { RRÇÃO MÉDIA } \\
(\mathrm{M}) \\
1 \text { barra pequena } \\
(25 \mathrm{~g})\end{array}$} & SUA & \multirow{2}{*}{$\begin{array}{c}\text { PORÇÃO } \\
\text { GE } \\
\square \square\end{array}$} \\
\hline $\begin{array}{c}\text { Chocolate, bombom, } \\
\text { brigadeiro }\end{array}$ & $\begin{array}{l}\text { N } 1 \\
\square \square\end{array}$ & 2 & $\begin{array}{l}3 \\
\square\end{array}$ & $\begin{array}{l}4 \\
\square\end{array}$ & $\begin{array}{l}5 \\
\square\end{array}$ & $\begin{array}{c}6 \\
\square\end{array}$ & $\begin{array}{l}7 \\
\square\end{array}$ & $\begin{array}{l}8 \\
\square\end{array}$ & $\begin{array}{l}9 \quad 10 \\
\square \quad \square\end{array}$ & $\mathrm{D}$ & $\mathrm{s}$ & $\begin{array}{l}\text { M A } \\
\square \square\end{array}$ & & $\begin{array}{l}\mathrm{P} \mathrm{M} \\
\square \square\end{array}$ & \\
\hline $\begin{array}{l}\text { Achocolatado em pó } \\
\text { (adicionado ao leite) }\end{array}$ & $\begin{array}{l}\text { N } 1 \\
\square \quad \square\end{array}$ & $\begin{array}{l}2 \\
\square\end{array}$ & $\begin{array}{l}3 \\
\square\end{array}$ & $\begin{array}{l}4 \\
\square\end{array}$ & $\begin{array}{l}5 \\
\square\end{array}$ & & $\begin{array}{l}7 \\
\square\end{array}$ & $\begin{array}{l}8 \\
\square\end{array}$ & & $\mathrm{D}$ & $\mathrm{s}$ & $\begin{array}{l}\text { M A } \\
\square \square\end{array}$ & $\begin{array}{c}2 \text { colheres de sopa } \\
(25 \mathrm{~g})\end{array}$ & $\begin{array}{l}\mathrm{P} \mathrm{M} \\
\square \quad \square\end{array}$ & $\begin{array}{l}\mathrm{GE} \\
\square \square\end{array}$ \\
\hline $\begin{array}{l}\text { Sobremesas, doces, } \\
\text { tortas e pudins }\end{array}$ & $\begin{array}{l}\text { N } 1 \\
\square \square\end{array}$ & $\begin{array}{l}2 \\
\square\end{array}$ & $\begin{array}{l}3 \\
\square\end{array}$ & 4 & $\begin{array}{l}5 \\
\square\end{array}$ & & $\begin{array}{l}7 \\
\square\end{array}$ & $\begin{array}{l}8 \\
\square\end{array}$ & $\begin{array}{l}910 \\
\square \quad \square\end{array}$ & $\mathrm{D}$ & S & $\begin{array}{l}\text { M A } \\
\square \square\end{array}$ & $\begin{array}{c}1 \text { pedaço ou } 1 \text { fatia } \\
\text { média }(60 \mathrm{~g})\end{array}$ & $\begin{array}{l}\mathrm{P} \mathrm{M} \\
\square \quad \square\end{array}$ & $\begin{array}{l}\mathrm{GE} \\
\square \square\end{array}$ \\
\hline Açúcar, mel, geléia & $\begin{array}{l}\text { N } 1 \\
\square \quad \square\end{array}$ & 2 & $\begin{array}{l}3 \\
\square\end{array}$ & 4 & $\begin{array}{l}5 \\
\square\end{array}$ & $\begin{array}{c}6 \\
\square\end{array}$ & $\begin{array}{l}7 \\
\square\end{array}$ & $\begin{array}{l}8 \\
\square\end{array}$ & $\begin{array}{l}910 \\
\square \quad \square\end{array}$ & $\mathrm{D}$ & S & $\begin{array}{l}\text { M A } \\
\square \square\end{array}$ & $\begin{array}{c}1 / 2 \text { colher de sopa } \\
(6 \mathrm{~g})\end{array}$ & $\begin{array}{l}\mathrm{P} \mathrm{M} \\
\square \quad \square\end{array}$ & $\begin{array}{l}\mathrm{GE} \\
\square \square\end{array}$ \\
\hline
\end{tabular}


2. Por favor, liste qualquer outro alimento ou preparação importante que você costuma comer ou beber pelo menosUMAVEZPORSEMANAquenãoforamcitadosaqui(porexemplo:

leite-decoco,outrostiposdecarnes, receitascaseiras,cremedeleite,leitecondensado,gelatinaeoutrosdoces, etc.).

\begin{tabular}{|l|l|l|}
\hline ALIMENTO & FREQUÊNCIA POR SEMANA & QUANTIDADE CONSUMIDA \\
\hline & & \\
\hline & & \\
\hline & & \\
\hline
\end{tabular}

6. Quando você come carne bovina ou suína, você costuma comer a gordura visível?
(1) nunca ouraramente
( 2 )algumasvezes
( 3 )sempre
( 9 ) nãosabe

7. Quando você come frango ou peru, você costuma comer a pele?
(1) nunca ouraramente
( 2 )algumasvezes
( 3 )sempre
( 9 ) nãosabe 


\section{APÊNDICE 2 \\ Termo de Consentimento Livre e Esclarecido - TCLE}

Estudo da prevalência dos fatores de risco da Síndrome Metabólica em uma instituição de nível superior

\section{Centro Universitário de Brasília - UniCEUB}

Pesquisador(a) responsável: Vanessa Carvalho Moreira

\section{Pesquisador(a) assistente: Hanid de Sousa Versiani e Luciene da Silva Guedes}

Você está sendo convidado(a) a participar do projeto de pesquisa acima citado. O texto abaixo apresenta todas as informações necessárias sobre o que estamos fazendo. Sua colaboração neste estudo será de muita importância para nós, mas se desistir a qualquer momento, isso não lhe causará prejuízo.

O nome deste documentoquevocêestálendoéTermodeConsentimento Livre eEsclarecido (TCLE).

Antesdedecidirsedesejaparticipar(delivreeespontâneavontade)vocêdeveráler ecompreendertodo oconteúdo.Ao final, caso decida participar, você será solicitadoaassináloereceberáumacópiadomesmo.

Antes de assinar, façaperguntassobretudooquenãotiverentendidobem. A equipe deste estudoresponderá às suasperguntas a qualquermomento (antes, duranteeapósoestudo).

\section{Naturezaeobjetivosdoestudo}

- O objetivoespecíficodesteestudoé avaliar os parâmetros de risco (glicemia, perfil lipídico, perfil alimentar, medidas antropométricas e pressão arterial) para o desenvolvimento da Síndrome Metabólica.

- Vocêestásendoconvidadoaparticiparexatamentepor ser estudante de curso da saúde no UniCEUB.

\section{Procedimentosdoestudo}

- Sua participaçãoconsisteem responder a um questionário de frequência alimentar, oferecer amostra de sangue e permitir a aferição da pressão e das medidas antropométricas.

- Os procedimentos são: preenchimento do questionário alimentar, coleta de sangue venoso para a realização de exames para determinar o nível glicêmico (Hemoglobina glicada) e o perfil lipídico (Triglicerideos, LDL, HDL, Colesterol total), aferição da pressão arterial e medidas antropométricas para a verificação do peso corporal, de altura e circunferência abdominal e do pescoço.

- Nãohaverá nenhumaoutraforma deenvolvimentoou comprometimento neste estudo.

- A pesquisa será realizada nas dependências do Labocien, localizado na Faculdade de Ciências da Educação e da Saúde - FACES do UniCEUB, bloco 9 - campus Asa Norte - SEPN 707/907, Brasília-DF.

\section{Riscosebenefícios}

- Esteestudo possui riscos mínimos, como o surgimento de hematoma no lugar da punção que desaparece em até sete dias.

- Medidaspreventivas como o treinamento dos responsáveis pela coleta serãotomadas. Para minimizarqualquerriscoouincômodo, após a coleta você deverá pressionar o local puncionado por aproximadamente cinco minutos e em caso de hematomas, colocar compressa de gelo.

- O participante deverá estar em Jejum e com roupa adequada para fazermos as medidas antropométricas. Mulheres devem estar de short de malha e top e homens de bermuda e sem camisa.

- Caso esse procedimento possa gerar algum tipo de constrangimento, vocênão precisarealizálo. 
- Com suaparticipação nesta pesquisa você poderá receber os resultados dos exames, além de contribuir paramaiorconhecimentosobre a Síndrome Metabólica e seus riscos para a saúde.

\section{Participação,recusaedireitodeseretirardoestudo}

- Sua participaçãoé voluntária. Vocênãoterá nenhum prejuízose nãoquiser participar.

- Você poderá se retirar desta pesquisa a qualquer momento, bastandoparaisso entraremcontatocomumdospesquisadoresresponsáveis.

- Conformeprevistopelasnormasbrasileirasde pesquisacoma participaçãode seres humanos,vocênão receberánenhumtipodecompensaçãofinanceirapelasua participaçãonesteestudo.

\section{Confidencialidade}

- Seusdadosserãomanuseadossomentepelospesquisadores enãoserápermitidoo acessoaoutraspessoas.

- Os dados e instrumentos utilizados ficarãoguardadossobaresponsabilidadedo pesquisador responsável Profa. Vanessa Carvalho Moreira comagarantiademanutenção do sigiloeconfidencialidade, e arquivados por um período de 5 anos; após esse tempo serão destruídos.

- Osresultadosdestetrabalho poderão serapresentados emencontrosourevistas científicas. Entretanto, elemostraráapenasosresultadosobtidoscomo umtodo, sem revelar seunome, instituiçãoa qualpertenceou qualquer informação que estejarelacionadacom suaprivacidade.

Se houver alguma consideração ou dúvida referente aos aspectos éticos da pesquisa, entre em contato com o ComitêdeÉticaemPesquisadoCentroUniversitáriodeBrasília -CEP/UniCEUB, que aprovou esta pesquisa, pelo telefone3966.1511 ou pelo e-mail cep.uniceub@uniceub.br.Também entre em contato para informar ocorrências irregulares ou danosas durante a sua participação no estudo.

Eu, RG

apósreceberaexplicaçãocompletadosobjetivosdoestudoedosprocedimentos envolvidos nesta pesquisaconcordovoluntariamenteemfazerpartedesteestudo.

Este Termo de Consentimento encontra-se impresso em duas vias, sendo que uma cópia será arquivada pelo pesquisador responsável, e a outra será fornecida ao senhor(a).

Brasília, de de

Participante

Pesquisador responsável: Vanessa Carvalho Moreira, telefoneinstitucional 3966-1573

Pesquisador assistente: Hanid de Sousa Versiani (61-81434584)

Pesquisador assistente: Luciene da Silva Guedes (61-92106182)

Endereço dos(as) responsável(eis) pela pesquisa (OBRIGATÓRIO):

Instituição: Centro Universitário de Brasília

Endereço: SEPN 707/907 - Bloco 9, sala 9002, térreo.

Bairro: /CEP/Cidade: Setor Universitário/70.790-075/Brasília

Telefones p/contato: (61) 3966-1573 
APÊNDICE 3

Estudo da prevalência dos fatores de risco da Síndrome Metabólica em uma instituição de nível superior

Avaliação Antropométrica:

Data:

Nome do participante:

Idade Atual:

Data de Nascimento:

Nome do Curso:

Altura:

m Peso atual:

kg Feminino ( ) Masculino ( )

\begin{tabular}{|l|l|}
\hline Jackson \& Pollock- 7 Dobras & Medidas: \\
\hline Diâmetro Fêmur: & \\
\hline Diâmetro do Rádio Ulnar: & \\
\hline Diâmetro do Umero Radial & \\
\hline Dobra Abdominal: & \\
\hline Dobra Medial da Coxa: & \\
\hline Dobra Axilar Média: & \\
\hline Dobra Subescapular: & \\
\hline Dobra Suprailiaca: & \\
\hline Dobra Toraxica (Peitoral): & \\
\hline Dobra Triciptal: & \\
\hline Circunferencia do Pescoço: & \\
\hline Circunferencia da Cintura: & \\
\hline Circunferencia do Torax: & \\
\hline Circunferencia da Panturilha: & \\
\hline Circunferencia do Braço: & \\
\hline \% adequação do CB C* & \\
\hline \% adequação de CMB ${ }^{\star \star}$ & \\
\hline
\end{tabular}

